\title{
Identification of Matrix Conditions that Give Rise to the Linear Coupling Resonances
}

\author{
C.J. Gardner
}

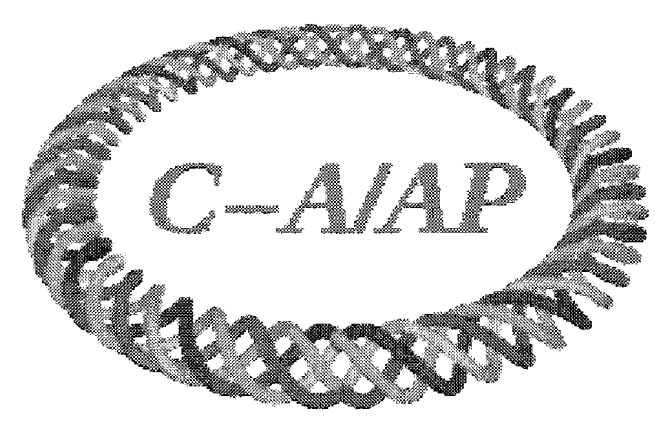

\section{Collider-Accelerator Department Brookhaven National Laboratory Upton, NY 11973}

Notice: This document has been authorized by employees of Brookhaven Science Associates, LLC under Contract No. DE-AC02-98CH10886 with the U.S. Department of Energy. The United States Government retains a nonexclusive, paid-up, irrevocable, world-wide license to publish or reproduce the published form of this document, or allow others to do so, for United States Government purposes. 


\title{
Identification of Matrix Conditions that Give Rise to the Linear Coupling Resonances
}

\author{
C.J. Gardner
}

Brookhaven Notional Laboratory, Upton, NY 11973, USA

\begin{abstract}
General definitions of horizontal and vertical amplitudes for linear coupled motion are developed from the normal form of the one-turn matrix. This leads to the identification of conditions on the matrix that give rise to the linear coupling sum and difference resonances. The correspondence with the standard hamiltonian treatment of the resonances is discussed.
\end{abstract}

\section{Introduction}

One of the hallmarks of linear coupling is the resonant exchange of oscillation amplitude between the horizontal and vertical planes when the difference between the unperturbed tunes is close to an integer. The standard derivation of this phenomenon (that is, the difference resonance) can be found, for example, in the classic papers of Guignard $[1,2,3]$. One starts with an uncoupled lattice and adds a linear perturbation that couples the two planes. The equations of motion are expressed in hamiltonian form. As the difference between the unperturbed tunes approaches an integer, one finds that the perturbing terms in the hamiltonian can be divided into terms that oscillate slowly and ones that oscillate rapidly. The rapidly oscillating terms are discarded or transformed to higher order with an appropriate canonical transformation. The resulting approximate hamiltonian gives equations of motion that clearly exhibit the exchange of oscillation amplitude between the two planes.

If, instead of the hamiltonian, one is given the four-by-four matrix for one turn around a synchrotron, then one has the complete solution for the turn-by-turn motion. However, the conditions for the phenomenon of amplitude exchange are not obvious from a casual inspection of the matrix. These conditions and those that give rise to the related sum resonance are identified in this article. The identification is made by expressing the one-turn

Email addresses: cgardner@bnl.gov (C.J. Gardner) $B N L C \sim A / A P$ Note matrix in normal form and defining appropriate amplitudes for oscillations in the horizontal and vertical planes. The resonance conditions are found to be encoded in a two-by-two matrix $\mathcal{M}$ formed from the normal-form matrix $\mathcal{W}$. The formulae obtained are general in that no particular hamiltonian or coupling elements are assumed. The only assumptions are that the one-turn matrix is symplectic and that it has distinct eigenvalues on the unit circle in the complex plane.

The outline of the article is as follows: In Section 2 the properties of the one-turn symplectic matrix are reviewed. In Sections 3 and 4 the normal form of the matrix and normalized coordinates are introduced. The matched ellipsoid and the horizontal and vertical amplitudes are introduced in Sections 5 and 6 . The conditions for resonance are identified and discussed in Sections 7 through 10 . In Section 11 the degrees of freedom in the choice of the normal-form matrix are examined and various parameters and matrices that are independent of this choice are identified. The Edwards-Teng parameters $[4,5,6]$ are derived from the normal-form matrix in Section 12 and the matrix $\mathcal{M}$ is shown to be proportional to the normalized coupling matrix of Sagan and Rubin [7]. If the conditions for one resonance dominate over those for the other, the number of parameters needed to specify the oneturn matrix is reduced from ten to eight. This is exploited in Section 13 where the measurement of coupling parameters for the case of the difference resonance is discussed. For completeness, the coupled lattice parameters introduced by Ripken $[8,9]$

Fcomary 15, 2009 
are derived from the normal-form matrix in Section 14.

\section{The One-Turn Matrix}

Let $X_{0}, X_{0}^{\prime}, Y_{0}, Y_{0}^{\prime}$ be the initial horizontal and vertical positions and angles of a beam particle at some point along the equilibrium orbit of a synchrotron, and let $X, X^{\prime}, Y, Y^{\prime}$ be the positions and angles at the point on the nth turn around the machine. Writing

$$
\mathbb{Z}=\left(\begin{array}{c}
X \\
X^{\prime} \\
Y \\
Y^{\prime}
\end{array}\right), \quad \mathbb{Z}_{0}=\left(\begin{array}{c}
X_{0} \\
X_{0}^{\prime} \\
Y_{0} \\
Y_{0}^{\prime}
\end{array}\right)
$$

we have

$$
\mathbf{Z}=\mathrm{T}^{r} \mathbf{Z}_{0}
$$

where

$$
\mathbf{T}=\left(\begin{array}{cccc}
T_{11} & T_{12} & T_{13} & T_{14} \\
T_{21} & T_{22} & T_{23} & T_{24} \\
T_{31} & T_{32} & T_{33} & T_{34} \\
T_{41} & T_{42} & T_{43} & T_{44}
\end{array}\right)
$$

is the four-by-four transfer matrix for one turn around the machine. It will be convenient to partition $\mathbf{Z}_{0}, \mathbf{Z}$ and $\mathbf{T}$ into two-component vectors and two-by-two matrices. Thus

$$
\begin{array}{ll}
\mathbf{Z}_{0}=\left(\begin{array}{c}
\mathbf{X}_{0} \\
\mathbf{Y}_{0}
\end{array}\right), & \mathbf{Z}=\left(\begin{array}{c}
\mathbf{X} \\
\mathbf{Y}
\end{array}\right) \\
\mathbf{X}_{0}=\left(\begin{array}{c}
X_{0} \\
X_{0}^{\prime}
\end{array}\right), & \mathbf{X}=\left(\begin{array}{c}
X \\
X^{\prime}
\end{array}\right) \\
\mathbf{Y}_{0}=\left(\begin{array}{c}
Y_{0} \\
Y_{0}^{\prime}
\end{array}\right), & \mathbf{Y}=\left(\begin{array}{c}
Y \\
Y^{\prime}
\end{array}\right)
\end{array}
$$

and

$$
\mathrm{T}=\left(\begin{array}{ll}
\mathrm{M} & \mathbf{n} \\
\mathrm{m} & \mathrm{N}
\end{array}\right)
$$

where

$$
\begin{aligned}
& \mathrm{M}=\left(\begin{array}{ll}
M_{11} & M_{12} \\
M_{21} & M_{22}
\end{array}\right) \\
& \mathbf{N}=\left(\begin{array}{ll}
N_{11} & N_{12} \\
N_{21} & N_{22}
\end{array}\right) \\
& \mathbf{m}=\left(\begin{array}{ll}
m_{11} & m_{12} \\
m_{21} & m_{22}
\end{array}\right)
\end{aligned}
$$

$$
\mathbf{n}=\left(\begin{array}{ll}
n_{11} & n_{12} \\
n_{21} & n_{22}
\end{array}\right)
$$

The matrix elements of $m$ and $n$ are proportional to the skew quadrupole or solenoidal felds that give rise to linear coupling between the horizontal and vertical planes of oscillation.

Following Cournt and Snyder [10], we define the symplectic conjugate of any two-by-two matrix

$$
\mathbf{A}=\left(\begin{array}{ll}
A_{11} & A_{12} \\
A_{21} & A_{22}
\end{array}\right)
$$

to be

$$
\overline{\mathbf{A}}=\left(\begin{array}{rr}
A_{22} & -A_{12} \\
-A_{21} & A_{11}
\end{array}\right) .
$$

We then have

$$
\overline{\mathbf{A}}=\overline{\mathbf{A}} \mathbf{A}=|\mathbf{A}| \mathbb{I}
$$

and

$$
\mathbf{A}+\overline{\mathbf{A}}=(\operatorname{Tr} \mathbf{A}) \mathbf{I}
$$

where

$$
\begin{aligned}
& |\mathbf{A}|=A_{11} A_{22}-A_{12} A_{21} \\
& \operatorname{Tr} \mathbf{A}=A_{11}+A_{22}
\end{aligned}
$$

and

$$
\mathbf{I}=\left(\begin{array}{ll}
1 & 0 \\
0 & 1
\end{array}\right)
$$

is the multiplicative identity.

The matrix $\mathbf{T}$ is symplectic and we assume that its four eigenvalues are distinct and lie on the unit circle in the complex plane. We assume further that none of the eigenvalues is equal to 1 or -1 . The four eigenvalues are then $\lambda_{1}, \lambda_{1}^{*}, \lambda_{2}$ and $\lambda_{2}^{*}$, where $[10]$

$$
\begin{aligned}
& \lambda_{1}=\exp i \psi_{1}, \quad \lambda_{2}=\exp i h_{2} \\
& 4 \cos \psi_{1}=M+N \pm \sqrt{T^{2}+4|\mathrm{~m}+\overline{\mathbf{n}}|} \\
& 4 \cos \psi_{2}=M+N \mp \sqrt{\left(T^{2}+4|\mathrm{~m}+\overline{\mathbf{n}}|\right.} .
\end{aligned}
$$

Here

$$
\begin{aligned}
& M=\operatorname{Tr} \mathrm{M}=M_{11}+M_{22} \\
& N=\operatorname{Tr} \mathrm{N}=N_{11}+N_{22}
\end{aligned}
$$

and

$$
T=\operatorname{Tr}(\mathbf{M}-\mathbf{N})=M-N .
$$


Under our assumptions, the phases $\psi_{1}$ and $\psi_{2}$ are real with $\cos \psi_{1} \neq \cos \psi_{2}$. The tunes associated with the eigenvalues are

$$
Q_{1}=\psi_{1} /(2 \pi), \quad Q_{2}=\psi_{2} /(2 \pi)
$$

and no element of the set $Q_{1}, 2 Q_{1}, Q_{2}, 2 Q_{2}, Q_{1}+$ $Q_{2}, Q_{1}-Q_{2}$ is equal to an integer. We also define

$$
U=2 \cos \psi_{1}-2 \cos \psi_{2}
$$

which gives

$$
U= \pm \sqrt{T^{2}+4|\mathbf{m}+\overline{\mathbf{n}}|}
$$

We shall adopt the convention (7) that signs in front of the square roots in $(20),(21)$ and (27) are chosen so the $U$ has the same sign as $T$.

The symplectic condition implies that the twoby-two submatrices of $T$ satisfy

$$
\begin{aligned}
|\mathbf{M}|+|\mathbf{m}| & =1 \\
|\mathbf{N}|+|\mathbf{n}| & =1 \\
\overline{\mathbf{M}} \mathbf{n}+\overline{\mathbf{m}} \mathbf{N} & =\mathbf{0}
\end{aligned}
$$

and

$$
\begin{aligned}
|\mathbf{M}|+|\mathbf{n}| & =1 \\
|\mathbf{N}|+|\mathbf{m}| & =1 \\
\mathbf{M} \overline{\mathbf{m}}+\mathbf{n} \overline{\mathbf{N}} & =\mathbf{0}
\end{aligned}
$$

where

$$
\mathbf{0}=:\left(\begin{array}{ll}
0 & 0 \\
0 & 0
\end{array}\right)
$$

Equations (28) and (29) are actually equivalent, and, as shown by Brown and Servranckx [11], they impose a total of 6 independent constraints on the 16 matrix elements of $\mathbf{T}$. The four-by-four symplectic matrix $\mathbf{T}$ is therefore specified by 10 independent parameters. Equations (28) and (29) also imply

$$
|\mathbf{M}|=|\mathbf{N}|, \quad|\mathbf{m}|=|\mathbf{n}| .
$$

\section{Normal Form}

Under our assumptions it is possible to write $\mathbf{T}$ in the form $[12,13]$

$$
\mathrm{T}=\mathcal{W U W}^{-1}
$$

where

$$
\mathcal{U}=\left(\begin{array}{cc}
\mathcal{A} & 0 \\
0 & \mathcal{B}
\end{array}\right)
$$

$$
\begin{aligned}
& \mathcal{A}=\left(\begin{array}{rr}
\cos \psi_{1} & \sin \psi_{1} \\
-\sin \psi_{1} & \cos \psi_{1}
\end{array}\right) \\
& \mathcal{B}=\left(\begin{array}{rr}
\cos \psi_{2} & \sin \psi_{2} \\
-\sin \psi_{2} & \cos \psi_{2}
\end{array}\right)
\end{aligned}
$$

and $\mathcal{W}$ is a four-by-four symplectic matrix. We call this nomal form. Here we derive some important relations between the elements of $T$ and those of $U$ and $\mathcal{W}$. Partitioning $\mathcal{W}$ into two-by-two matrices we have

$$
W=\left(\begin{array}{ll}
W_{1} & \mathcal{D}_{1} \\
\mathcal{D}_{2} & \mathcal{W}_{2}
\end{array}\right)
$$

and the symplectic condition implies

$$
\begin{aligned}
\left|\mathcal{W}_{1}\right|+\left|\mathcal{D}_{2}\right| & =1 \\
\left|\mathcal{W}_{2}\right|+\left|\mathcal{D}_{1}\right| & =1 \\
\bar{W}_{1} \mathcal{D}_{1}+\overline{\mathcal{D}}_{2} \mathcal{W}_{2} & =0 \\
\left|\mathcal{W}_{1}\right|+\left|\mathcal{D}_{1}\right| & =1 \\
\left|\mathcal{W}_{2}\right|+\left|\mathcal{D}_{2}\right| & =1 \\
\mathcal{W}_{1} \overline{\mathcal{D}}_{2}+\mathcal{D}_{1} \overline{\mathcal{W}}_{2} & =0 \\
\left|\mathcal{W}_{1}\right|=\left|\mathcal{W}_{2}\right|, \quad\left|\mathcal{D}_{1}\right| & =\left|\mathcal{D}_{2}\right|
\end{aligned}
$$

and

$$
\mathcal{W}^{-1}=\left(\begin{array}{ll}
\overline{\mathcal{W}}_{1} & \overline{\mathcal{D}}_{2} \\
\overline{\mathcal{D}}_{1} & \overline{\mathcal{W}}_{2}
\end{array}\right) .
$$

Thus we have

$$
\begin{gathered}
\left(\begin{array}{cc}
\mathbf{M} & \mathbf{n} \\
\mathbf{m} & \mathbf{N}
\end{array}\right)= \\
\left(\begin{array}{cc}
\mathcal{W}_{1} & \mathcal{D}_{1} \\
\mathcal{D}_{2} & \mathcal{W}_{2}
\end{array}\right)\left(\begin{array}{cc}
\mathcal{A} & \mathbf{0} \\
0 & \mathcal{B}
\end{array}\right)\left(\begin{array}{ll}
\overline{\mathcal{W}}_{1} & \overline{\mathcal{D}}_{2} \\
\overline{\mathcal{D}}_{1} & \overline{\mathcal{W}}_{2}
\end{array}\right)
\end{gathered}
$$

which gives

$$
\begin{aligned}
& \mathbf{M}=\mathcal{W}_{1} \mathcal{A} \overline{\mathcal{W}}_{1}+\mathcal{D}_{1} \mathcal{B} \overline{\mathcal{D}}_{1} \\
& \mathrm{~N}=\mathcal{D}_{2} \mathcal{A} \overline{\mathcal{D}}_{2}+\mathcal{W}_{2} \mathcal{B} \overline{\mathcal{W}}_{2} \\
& \mathbf{m}=\mathcal{D}_{2} \mathcal{A} \overline{\mathcal{W}}_{1}+\mathcal{W}_{2} \mathcal{B \mathcal { D }} 1 \\
& \mathbf{n}=\mathcal{W}_{1} \mathcal{A} \overline{\mathcal{D}}_{2}+\mathcal{D}_{1} \mathcal{B} \overline{\mathcal{W}}_{2}
\end{aligned}
$$

Taking the trace of (42) and (43) gives

$$
\begin{aligned}
& M=D \operatorname{Tr} \mathcal{A}+(1-D) \operatorname{Tr} \mathcal{B} \\
& N=(1-D) \operatorname{Tr} \mathcal{A}+D \operatorname{Tr} \mathcal{B} \\
& M-N=(2 D-1) \operatorname{Tr}(\mathcal{A}-\mathcal{B})
\end{aligned}
$$


where

$$
\begin{aligned}
& D=\left|\mathcal{W}_{1}\right|=\left|\mathcal{W}_{2}\right| \\
& 1-D=\left|\mathcal{D}_{2}\right|=\left|\mathcal{D}_{1}\right| .
\end{aligned}
$$

Thus using (24) and (26) we have

$$
T=(2 D-1) U
$$

and since we have adopted the convention that the signs in (20) and (21) are chosen so that $U$ las the same sign as $T$, we must have

$$
D \geq 1 / 2 \text {. }
$$

Taking the symplectic conjugate of (45) gives

$$
\overline{\mathbf{n}}=\mathcal{D}_{2} \overline{\mathcal{A}} \bar{W}_{1}+\mathcal{W}_{2} \overline{\mathcal{B}} \overline{\mathcal{D}}_{1}
$$

and therefore

$$
\mathbf{m}+\overline{\mathbf{n}}=\mathcal{D}_{2} \overline{\mathcal{W}}_{1} \operatorname{Tr} \mathcal{A}+\mathcal{W}_{2} \overline{\mathcal{D}}_{1} \operatorname{Tr} \mathcal{B}
$$

Using (38) we then have

$$
\mathbf{m}+\overline{\mathbf{n}}=U \mathcal{D}_{2} \overline{\mathcal{W}}_{1}
$$

and

$$
|\mathbf{m}+\overline{\mathbf{n}}|=U^{2} D(1-D) .
$$

Note that this follows aiso from (51) and (27).

\section{Normalized Coordinates}

Using (32) in (2) we have

$$
\mathbf{Z}=\mathcal{W U}^{n} \mathcal{W}^{-1} \mathbf{Z}_{0}
$$

and multiplying by $\mathcal{W}^{-1}$ from the left we see that it is natural to introduce normalized coordinates [14] defined by

$$
\begin{aligned}
& \widehat{\mathbf{Z}}=\mathcal{W}^{-1} \mathbf{Z} \\
& \widehat{\mathbf{Z}}_{0}=\mathcal{W}^{-1} \mathbf{Z}_{0} .
\end{aligned}
$$

This gives

$$
\widehat{\mathbf{Z}}=\mathcal{U}^{n} \widehat{\mathbf{Z}}_{0} .
$$

Partitioning $\widehat{\mathbf{Z}}$ and $\widehat{\mathrm{Z}}_{0}$ into two-component vectors we have

$$
\hat{\mathbf{Z}}=\left(\begin{array}{c}
\hat{\mathbf{X}} \\
\hat{\mathbf{Y}}
\end{array}\right), \quad \widehat{\mathbf{Z}}_{0}=\left(\begin{array}{c}
\widehat{\mathbf{X}}_{0} \\
\widehat{\mathbf{Y}}_{0}
\end{array}\right)
$$

and (60) becomes

$$
\widehat{\mathbf{X}}=\mathcal{A}^{n} \widehat{\mathbf{X}}_{0}, \quad \widehat{\mathbf{Y}}=\mathcal{B}^{n} \widehat{\mathbf{Y}}_{0}
$$

where

$$
\begin{aligned}
& \hat{\mathbf{X}}=\left(\begin{array}{c}
\hat{X} \\
\hat{X}^{\prime}
\end{array}\right), \quad \widehat{\mathbf{Y}}=\left(\begin{array}{c}
\widehat{Y} \\
\hat{Y}^{\prime}
\end{array}\right) \\
& \widehat{\mathbf{X}}_{0}=\left(\begin{array}{c}
\hat{X}_{0} \\
\hat{X}_{0}^{\prime}
\end{array}\right), \quad \hat{\mathbf{Y}}_{0}=\left(\begin{array}{c}
\hat{Y}_{0} \\
\widehat{Y}_{0}^{\prime}
\end{array}\right) .
\end{aligned}
$$

Then using the identities

$$
\left(\mathcal{A}^{n}\right)^{\dagger} \mathcal{A}^{n}=\mathbf{I} . \quad\left(\mathcal{B}^{n}\right)^{\dagger} \mathcal{B}^{n}=\mathbf{I}
$$

we have

$$
\widehat{\mathbf{X}}^{\dagger} \hat{\mathbf{X}}=\widehat{\mathbf{X}}_{0}^{\dagger} \widehat{\mathbf{X}}_{0}=\epsilon_{1}
$$

and

$$
\widehat{\mathbf{Y}}^{\dagger} \widehat{\mathbf{Y}}=\hat{\mathbf{Y}}_{0}^{\dagger} \widehat{\mathbf{Y}}_{0}=\epsilon_{2}
$$

where

$$
\begin{array}{ll}
\hat{\mathbf{X}}^{\dagger} \widehat{\mathbf{X}}=\hat{X}^{2}+\hat{X}^{\prime 2}, & \widehat{\mathbf{X}}_{0}^{\dagger} \widehat{\mathbf{X}}_{0}=\hat{X}_{0}^{2}+\hat{X}_{0}^{\prime 2} \\
\hat{\mathbf{Y}}^{\dagger} \hat{\mathbf{Y}}=\widehat{Y}^{2}+\widehat{Y}^{\prime 2}, & \widehat{\mathbf{Y}}_{0}^{\dagger} \widehat{\mathbf{Y}}_{0}=\widehat{Y}_{0}^{2}+\widehat{Y}_{0}^{\prime 2} .
\end{array}
$$

Here and throughout the text we use a dagger to denote the transpose of a vector or matrix. These equations define the normal-mode emittances, $\epsilon_{1}$ and $\epsilon_{2}$, and show that they are conserved quantities. They also show that there are phases $\phi_{1}$ and $\phi_{2}$ such that

$$
\begin{array}{ll}
\widehat{X}_{0}=\sqrt{\epsilon_{1}} \cos \phi_{1}, & \widehat{X}_{0}^{\prime}=\sqrt{\epsilon_{1}} \sin \phi_{1} \\
\widehat{Y}_{0}=\sqrt{\epsilon_{2}} \cos \phi_{2}, & \widehat{Y}_{0}^{\prime}=\sqrt{\epsilon_{2}} \sin \phi_{2} .
\end{array}
$$

The four parameters $\epsilon_{1}, \epsilon_{2}, \phi_{1}$ and $\phi_{2}$ are the "action-angle" parameters introduced by Luo [14]. These are initial condition parameters which either determine or are determined by $X_{0}, X_{0}^{\prime}, Y_{0}$ and $Y_{0}^{\prime}$ through equations (59) and (70-71).

Now writing (58) as

$$
\mathbf{z}=\mathcal{W} \widehat{\mathbf{Z}}
$$

and using (36) we have

$$
\begin{aligned}
& \mathbf{X}=\mathcal{W}_{1} \widehat{\mathbf{X}}+\mathcal{D}_{1} \widehat{\mathbf{Y}} \\
& \mathbf{Y}=\mathcal{D}_{2} \widehat{\mathbf{X}}+\mathcal{W}_{2} \hat{\mathbf{Y}} .
\end{aligned}
$$

Then using (62) we have

$$
\begin{aligned}
& \mathbf{X}=\mathcal{W}_{1} \mathcal{A}^{n} \widehat{\mathbf{X}}_{0}+\mathcal{D}_{1} \mathcal{B}^{n} \widehat{\mathbf{Y}}_{0} \\
& \mathbf{Y}=\mathcal{D}_{2} \mathcal{A}^{n} \widehat{\mathbf{X}}_{0}+\mathcal{W}_{2} \mathcal{B}^{n} \widehat{\mathbf{Y}}_{0}
\end{aligned}
$$

which give the turn-by-turn positions and angles $X$, $X^{\prime}, Y$ and $Y^{\prime}$ in terms of the submatrices of $\mathcal{W}$ and $\mathcal{U}$ and the initial normalized coordinates $\widehat{X}_{0}, \widehat{X}_{0}^{\prime}$, $\widehat{Y}_{0}$ and $\widehat{Y}_{0}^{\prime}$. 


\section{The Matched Ellipsoid}

Consider now the matrix

$$
\mathbf{E}=\mathcal{W} w^{\dagger} .
$$

Using (32) one finds that

$$
\mathbb{T E T}^{\dagger}=W Z \mathcal{U}^{\dagger} \mathcal{W}^{\dagger}
$$

and since $2 Z^{\dagger}=I$ we have

$$
\operatorname{TET}^{\dagger}=\mathbb{E} \text {. }
$$

By construction the matrix $\mathrm{E}$ has unit determinant and is real, symmetric and positive definite. (A real symmetric matrix $\mathbf{E}$ is positive definite if and only if the quadratic form $Z^{\dagger} \mathbf{E Z}>0$ for every vector $\mathbf{Z} \neq 0$.) It follows that the set of initial positions and angies $X_{0}, X_{0}^{\prime}, Y_{0}, Y_{0}^{\prime}$ defined by

$$
\mathbf{Z}_{0}^{\dagger} \mathbf{E}^{-1} \mathbf{Z}_{0}=\epsilon
$$

is a four dimensional ellipsoid. On the $n$th turn around the machine we have

$$
\mathbf{Z}=\mathbf{T}^{n} \mathbf{Z}_{0}
$$

and

$$
\mathbf{Z}^{\dagger} \mathbf{E}^{-1} \mathbf{Z}=\mathbf{Z}_{0}^{\dagger}\left(\mathbf{T}^{\dagger}\right)^{n} \mathbf{E}^{-1} \mathbf{T}^{n} \mathbf{Z}_{0} .
$$

But (79) implies

$$
\mathbf{T}^{\dagger} \mathbf{E}^{-1} \mathbf{T}=\mathbf{E}^{-1}
$$

and (by induction)

$$
\left(\mathbf{T}^{\dagger}\right)^{n} \mathbf{E}^{-1} \mathbf{T}^{n}=\mathbf{E}^{-1} .
$$

Thus

$$
\mathbf{Z}^{\dagger} \mathbf{E}^{-1} \mathbf{Z}=\mathbf{Z}_{0}^{\dagger} \mathbf{E}^{-1} \mathbf{Z}_{0}=\epsilon
$$

and we see that the particle positions and angles lie on the same ellipsoid after each turn. The ellipsoid is then said to be matched to the lattice.

\section{Horizontal and Vertical Amplitudes}

Partitioning $\mathbf{E}$ into two-by-two matrices and using (36) in (77) we have

$$
\mathbb{E}=\left(\begin{array}{ll}
\mathbf{F} & \mathbf{C} \\
\mathbf{C}^{\dagger} & \mathbf{G}
\end{array}\right)
$$

where

$$
\mathbb{F}=\mathcal{W}_{1} \mathcal{W}_{1}^{\dagger}+\mathcal{D}_{1} \mathcal{D}_{1}^{\dagger}
$$

$$
\begin{aligned}
& \mathbf{G}=\mathcal{W}_{2} \mathcal{W}_{2}^{\dagger}+\mathcal{D}_{2} \mathcal{D}_{2}^{\dagger} \\
& \mathbf{C}=\left\{\mathcal{W}_{1} \mathcal{D}_{2}^{\dagger}+\mathcal{D}_{1} \mathcal{W}_{2}^{\dagger}\right\}
\end{aligned}
$$

The projections of the matched ellipsoid onto the $X, X^{\prime}$ and $Y, Y^{\prime}$ planes are then the regions dofined by [11]

$$
\left\{\mathbf{X}^{\dagger} \mathbf{F}^{-1} \mathbf{X}\right\} \leq \epsilon
$$

and

$$
\left\{\mathbf{Y}^{\dagger} \mathbf{G}^{-1} \mathbf{Y}\right\} \leq t
$$

respectively. The borders of these regions are the ellipses defined by taking the equal signs in (90) and (91). This suggests that we define horizontal and vertical amplitudes

$$
J_{x}=F^{-1}\left\{\mathbf{X}^{\dagger} \mathbf{F}^{-1} \mathbf{X}\right\}=F\left\{\mathbf{X}^{\dagger} F \mathbf{X}\right\}
$$

and

$$
J_{y}=G^{-1}\left\{\mathbf{Y}^{\dagger} \mathbf{G}^{-1} \mathbf{Y}\right\}=G\left\{\mathbf{Y}^{\dagger} \overline{\mathbf{G}} \mathbf{Y}\right\}
$$

respectively, where

$$
F=|\mathbf{F}|^{-1 / 2}, \quad G=|\mathbf{G}|^{-1 / 2}
$$

and we have used the identities

$$
\mathbf{F}^{-1}=|\mathbf{F}|^{-1} \overline{\mathbf{F}}, \quad \mathbf{G}^{-1}=|\mathbf{G}|^{-1} \overline{\mathbf{G}} .
$$

Note that by construction the matrices $F \mathbf{F}$ and $G \mathbf{G}$ have unit determinant and are symmetric and positive definite. Thus we can write

$$
\begin{aligned}
& F \mathbf{F}=\left(\begin{array}{rr}
b_{x} & -a_{x} \\
-a_{x} & g_{x}
\end{array}\right) \\
& G \mathbf{G}=\left(\begin{array}{rr}
b_{y} & -a_{y} \\
-a_{y} & g_{y}
\end{array}\right)
\end{aligned}
$$

and we find that $J_{x}$ and $J_{y}$ have the familiar Courant-Snyder forms

$$
\begin{aligned}
& J_{x}=g_{x} X^{2}+2 a_{x} X X^{\prime}+b_{x} X^{\prime 2} \\
& J_{y}=g_{y} Y^{2}+2 a_{y} Y Y^{\prime}+b_{y} Y^{\prime 2}
\end{aligned}
$$

where

$$
b_{x} g_{x}-a_{x}^{2}=1, \quad b_{y} g_{y}-a_{y}^{2}=1 .
$$

To obtain $F$ and $G$ we need to calculate 


$$
\begin{gathered}
\mathrm{G} \overline{\mathbf{G}}= \\
\left\{\mathcal{W}_{2} \mathcal{W}_{2}^{\dagger}+\mathcal{D}_{2} \mathcal{D}_{2}^{\dagger}\right\}\left\{\overline{\mathcal{W}}_{2}^{\dagger} \overline{\mathcal{W}}_{2}+\overline{\mathcal{D}}_{2}^{\dagger} \overline{\mathcal{D}}_{2}\right\} .
\end{gathered}
$$

Here it will be useful to introduce the matrix

$$
\mathcal{M}=\overline{\mathcal{D}}_{1} \mathcal{W}_{1}=-\overline{\mathcal{W}}_{2} \mathcal{D}_{2}
$$

where the second equality follows from (37). Using (14-15) and (49-50) we have

$$
\begin{gathered}
\text { F } \overline{\mathbf{F}}= \\
\left\{D^{2}+(1-D)^{2}+\operatorname{Tr}\left(\mathcal{W}_{1} \mathcal{W}_{1}^{\dagger} \overline{\mathcal{D}}_{1}^{\dagger} \overline{\mathcal{D}}_{1}\right)\right\} \mathbf{I} \\
\text { G } \overline{\mathbf{G}}= \\
\left\{D^{2}+(1-D)^{2}+\operatorname{Tr}\left(\mathcal{D}_{2} \mathcal{D}_{2}^{\dagger} \overline{\mathcal{W}}_{2}^{\dagger} \overline{\mathcal{W}}_{2}\right)\right\} \mathbf{I}
\end{gathered}
$$

and using (103) we have

$$
\begin{aligned}
& \operatorname{Tr}\left(\mathcal{W}_{1} \mathcal{W}_{1}^{\dagger} \overline{\mathcal{D}}_{1}^{\dagger} \overline{\mathcal{D}}_{1}\right)=\operatorname{Tr}\left(\mathcal{M} \mathcal{M}^{\dagger}\right) \\
& \operatorname{Tr}\left(\mathcal{D}_{2} \mathcal{D}_{2}^{\dagger} \overline{\mathcal{W}}_{2}^{\dagger} \overline{\mathcal{W}}_{2}\right)=\operatorname{Tr}\left(\mathcal{M} \mathcal{M}^{\dagger}\right)
\end{aligned}
$$

Thus

$$
\begin{gathered}
F=G= \\
\left\{D^{2}+(1-D)^{2}+\operatorname{Tr}\left(\mathcal{M} \mathcal{M}^{\dagger}\right)\right\}^{-1 / 2}
\end{gathered}
$$

Now using (73) and (74) in (92) and (93) we have

$$
\begin{gathered}
J_{x}= \\
F\left\{\widehat{\mathbf{X}}^{\dagger} \mathcal{W}_{1}^{\dagger}+\widehat{\mathbf{Y}}^{\dagger} \mathcal{D}_{1}^{\dagger}\right\} \overline{\mathbf{F}}\left\{\mathcal{W}_{1} \widehat{\mathbf{X}}+\mathcal{D}_{1} \widehat{\mathbf{Y}}\right\} \\
J_{y}= \\
G\left\{\widehat{\mathbf{X}}^{\dagger} \mathcal{D}_{2}^{\dagger}+\widehat{\mathbf{Y}}^{\dagger} \mathcal{W}_{2}^{\dagger}\right\} \overline{\mathbf{G}}\left\{\mathcal{D}_{2} \widehat{\mathbf{X}}+\mathcal{W}_{2} \widehat{\mathbf{Y}}\right\}
\end{gathered}
$$

where

$$
\begin{aligned}
& \overline{\mathbf{F}}=\overline{\mathcal{W}}_{1}^{\dagger} \overline{\mathcal{W}}_{1}+\overline{\mathcal{D}}_{1}^{\dagger} \overline{\mathcal{D}}_{1} \\
& \mathcal{W}_{1}^{\dagger} \overline{\mathbf{F}} \mathcal{W}_{1}=D^{2} \mathbb{I}+\mathcal{M}^{\dagger} \mathcal{M} \\
& \mathcal{D}_{1}^{\dagger} \overline{\mathbf{F}} \mathcal{D}_{1}=(1-D)^{2} \mathbb{I}+\overline{\mathcal{M}}^{\dagger} \overline{\mathcal{M}} \\
& \mathcal{W}_{1}^{\dagger} \overline{\mathbf{F}} \mathcal{D}_{1}=D \overline{\mathcal{M}}+(1-D) \mathcal{M}^{\dagger}
\end{aligned}
$$

and

$$
\begin{aligned}
& \overline{\mathbf{G}}=\overline{\mathcal{W}}_{2}^{\dagger} \overline{\mathcal{W}}_{2}+\overline{\mathcal{D}}_{2}^{\dagger} \overline{\mathcal{D}}_{2} \\
& \mathcal{D}_{2}^{\dagger} \overline{\mathbf{G}} \mathcal{D}_{2}=(1-D)^{2} \mathbf{I}+\mathcal{M}^{\dagger} \mathcal{M} \\
& \mathcal{W}_{2}^{\dagger} \overline{\mathbf{G}} \mathcal{W}_{2}=D^{2} \mathbf{I}+\overline{\mathcal{M}}^{\dagger} \overline{\mathcal{M}}
\end{aligned}
$$

$$
\mathcal{D}_{2}^{\dagger} \overline{\mathbf{G}} \mathcal{W}_{2}=-(1-D) \overline{\mathcal{M}}-D \mathcal{M}^{\dagger}
$$

Thus

$$
\begin{array}{r}
J_{x}=F\left\{D^{2} \epsilon_{1}+(1-D)^{2} \epsilon_{2}\right\} \\
+F\left\{\hat{\mathbf{X}}^{\dagger} \mathcal{M}^{\dagger} \mathcal{M} \hat{\mathbf{X}}+\hat{\mathbf{Y}}^{\dagger} \overline{\mathcal{M}}^{\dagger} \overline{\mathcal{M}} \hat{\mathbf{Y}}\right\} \\
+2 F\left\{D \hat{\mathbf{X}}^{\dagger} \overline{\mathcal{M}} \hat{\mathbf{Y}}+(1-D) \hat{\mathbf{X}}^{\dagger} \mathcal{M}^{\dagger} \widehat{\mathbf{Y}}\right\} \\
J_{z}=F\left\{(1-D)^{2} \epsilon_{1}+D^{2} \epsilon_{2}\right\} \\
+F\left\{\hat{\mathbf{X}}^{\dagger} \mathcal{M}^{\dagger} \mathcal{M} \hat{\mathbf{X}}+\widehat{\mathbf{Y}}^{\dagger} \overline{\mathcal{M}}^{\dagger} \overline{\mathcal{M}} \hat{\mathbf{Y}}\right\} \\
-2 F\left\{(1-D) \hat{\mathbf{X}}^{\dagger} \overline{\mathcal{M}} \hat{\mathbf{Y}}+D \hat{\mathbf{X}}^{\dagger} \mathcal{M}^{\dagger} \hat{\mathbf{Y}}\right\}
\end{array}
$$

where $F$ is given by (108).

Note that we can use (103) to express $\mathcal{D}_{1}$ and $\mathcal{D}_{2}$ in terms of $\mathcal{W}_{1}, \mathcal{W}_{2}$ and $\mathcal{M}$. Thus

$$
\mathcal{D}_{1}=\frac{1}{D} \mathcal{W}_{1} \overline{\mathcal{M}}, \quad \mathcal{D}_{2}=-\frac{1}{D} \mathcal{W}_{2} \mathcal{M}
$$

and we have

$$
\begin{aligned}
& \mathcal{D}_{1} \mathcal{D}_{1}^{\dagger}=\frac{1}{D^{2}} \mathcal{W}_{1} \overline{\mathcal{M}} \overline{\mathcal{M}}^{\dagger} \mathcal{W}_{1}^{\dagger} \\
& \mathcal{D}_{2} \mathcal{D}_{2}^{\dagger}=\frac{1}{D^{2}} \mathcal{W}_{2} \mathcal{M} \mathcal{M}^{\dagger} \mathcal{W}_{2}^{\dagger} \\
& \mathcal{W}_{1} \mathcal{D}_{2}^{\dagger}=-\frac{1}{D} \mathcal{W}_{1} \mathcal{M}^{\dagger} \mathcal{W}_{2}^{\dagger} \\
& \mathcal{D}_{1} \mathcal{W}_{2}^{\dagger}=\frac{1}{D} \mathcal{W}_{1} \overline{\mathcal{M}} \mathcal{W}_{2}^{\dagger}
\end{aligned}
$$

This gives

$$
\begin{aligned}
& \mathbf{F}=\mathcal{W}_{1}\left\{\mathbf{I}+\frac{1}{D^{2}} \overline{\mathcal{M}} \overline{\mathcal{M}} \bar{W}^{\dagger}\right\} \mathcal{W}_{1}^{\dagger} \\
& \mathbf{G}=\mathcal{W}_{2}\left\{\mathbf{I}+\frac{1}{D^{2}} \mathcal{M} \mathcal{M}^{\dagger}\right\} \mathcal{W}_{2}^{\dagger} \\
& \mathbf{C}=\frac{1}{D} \mathcal{W}_{1}\left\{\overline{\mathcal{M}}-\mathcal{M}^{\dagger}\right\} \mathcal{W}_{2}^{\dagger} .
\end{aligned}
$$

\section{Sum and Difference of the Amplitudes}

Taking the sum and difference of (119) and (120) we have

$$
\begin{aligned}
J_{x}+J_{y} & =F\left\{D^{2}+(1-D)^{2}\right\}\left(\epsilon_{1}+\epsilon_{2}\right) \\
+ & 2 F\left\{\hat{\mathbf{X}}^{\dagger} \mathcal{M}^{\dagger} \mathcal{M} \hat{\mathbf{X}}+\widehat{\mathbf{Y}}^{\dagger} \overline{\mathcal{M}}^{\dagger} \overline{\mathcal{M}} \hat{\mathbf{Y}}\right\} \\
+ & 2 F(2 D-1) \hat{\mathbf{X}}^{\dagger}\left\{\overline{\mathcal{M}}-\mathcal{M}^{\dagger}\right\} \hat{\mathbf{Y}}
\end{aligned}
$$


and

$$
\begin{aligned}
J_{x}-J_{y} & =F^{\prime}(2 D-1)\left(\epsilon_{1}-\epsilon_{2}\right) \\
& +2 F \widehat{\mathbf{X}}^{\dagger}\left\{\bar{M}+M^{\dagger}\right\} \hat{\mathbf{Y}}
\end{aligned}
$$

where $M$ is given by (103) and

$$
\overline{M M}=\overline{\mathcal{D}}_{1} \mathcal{W}_{1} \overline{\mathcal{W}}_{1} \mathcal{D}_{1}=D(1-D) \mathrm{I} .
$$

Here we see that if

$$
\bar{M}=\mathcal{M}^{\dagger}
$$

then

$$
\begin{aligned}
& \overline{\mathcal{M}}^{\dagger} \overline{\mathcal{M}}=\widehat{M} \bar{M}=D(1-D) \mathbb{I} \\
& \mathcal{M}^{\dagger} \mathcal{M}=\bar{M} \mathcal{M}=D(1-D) \mathbb{I} \\
& \operatorname{Tr}\left(\mathcal{M M}^{\dagger}\right)=\operatorname{Tr}\left(\mathcal{M}^{\dagger} \mathcal{M}\right)=2 D(1-D) \\
& \begin{aligned}
F & =\left\{D^{2}+(1-D)^{2}+2 D(1-D)\right\}^{-1 / 2} \\
& =1
\end{aligned}
\end{aligned}
$$

and (129) becomes

$$
J_{x}+J_{y}=\epsilon_{1}+\epsilon_{2} .
$$

This is just the result obtained in the standard hamilonian treatment $[1,2,3]$ of the linear coupling difference resonance. Under the condition (132) we also find that $(126),(127)$ and (128) become

$$
\begin{aligned}
& \mathbf{F}=\frac{1}{D} \mathcal{W}_{1} \mathcal{W}_{1}^{\dagger}, \quad \mathbf{G}=\frac{1}{D} \mathcal{W}_{2} \mathcal{W}_{2}^{\dagger} \\
& \mathbf{C}=\mathbf{0} .
\end{aligned}
$$

We shall see in Section 12 that the elements of $\mathcal{W}_{1} \mathcal{W}_{1}^{\dagger} / D$ and $\mathcal{W}_{2} \mathcal{W}_{2}^{\dagger} / D$ are just the CourantSnyder parameters introduced by Edwards and Teng.

Similarly, if

$$
\overline{\mathcal{M}}=-\mathcal{M}^{\dagger}
$$

then

$$
\begin{aligned}
& \mathcal{M}^{\dagger} \mathcal{M}=-\overline{\mathcal{M}} \mathcal{M}=-D(1-D) \mathbf{I} \\
& \operatorname{Tr}\left(\mathcal{M M}^{\dagger}\right)=\operatorname{Tr}\left(\mathcal{M}^{\dagger} \mathcal{M}\right)=-2 D(1-D) \\
& \begin{aligned}
F= & =\left\{D^{2}+(1-D)^{2}-2 D(1-D)\right\}^{-1 / 2} \\
& =(2 D-1)^{-1}
\end{aligned}
\end{aligned}
$$

and (130) becomes

$$
J_{x}-J_{y}=\epsilon_{1}-\epsilon_{2}
$$

which is just the result obtained in the standard treatment of the linear coupling sum resonance. Under the condition (140) we also find that (126) and (127) become

$$
\begin{aligned}
& \mathrm{F}=(2 D-1) \frac{\mathcal{W}_{1} \mathcal{W}_{1}^{\dagger}}{D} \\
& \mathrm{G}=(2 D-1) \frac{\mathcal{W}_{2} \mathcal{W}_{2}^{\dagger}}{D}
\end{aligned}
$$

and we again find that $F$ and $G$ are proportional to $\mathcal{W}_{1} W_{1}^{\dagger} D$ and $\mathcal{W}_{2} \mathcal{W}_{2}^{\dagger} / D$

\section{Further Algebraic Reduction of the Am- plitudes}

Let us now look more closely at the matrix $\mathcal{M}$ which appears prominently in the expressions for the amplitudes. Writing

$$
\mathcal{M}=\left(\begin{array}{ll}
\mathcal{M}_{11} & \mathcal{M}_{12} \\
\mathcal{M}_{21} & \mathcal{M}_{22}
\end{array}\right)
$$

it is useful [7] to define parameters $A, B, \omega$, and $\psi$ such that.

$$
\begin{aligned}
& 2 A \cos \omega=\mathcal{M}_{11}+\mathcal{M}_{22} \\
& 2 A \sin \omega=\mathcal{M}_{12}-\mathcal{M}_{21} \\
& 2 B \cos \psi=\mathcal{M}_{11}-\mathcal{M}_{22} \\
& 2 B \sin \psi=\mathcal{M}_{12}+\mathcal{M}_{21} .
\end{aligned}
$$

This shows that $\mathcal{M}$ can always be written in the form [7]

$$
\mathcal{M}=A \Omega+B \Psi
$$

where

$$
\begin{aligned}
& \Omega=\left(\begin{array}{rr}
\cos \omega & \sin \omega \\
-\sin \omega & \cos \omega
\end{array}\right) \\
& \Psi=\left(\begin{array}{rr}
\cos \psi & \sin \psi \\
\sin \psi & -\cos \psi
\end{array}\right) .
\end{aligned}
$$

The matrices $\Omega$ and $\Psi$ have the properties

$$
\begin{aligned}
& \Omega \Omega^{\dagger}=\Omega^{\dagger} \Omega=\Psi \Psi^{\dagger}=\Psi^{\dagger} \Psi=I \\
& \bar{\Omega}=\Omega^{\dagger}, \quad \bar{\Psi}=-\Psi, \quad \Psi^{\dagger}=\Psi .
\end{aligned}
$$


Thus

$$
\begin{aligned}
& \mathcal{M}^{\dagger}=A \Omega^{\dagger}+B \Psi \\
& \bar{M}=A \Omega^{\dagger}-B \Psi
\end{aligned}
$$

and we see that

$$
\bar{M}-M^{\dagger}=0
$$

if and only if $B=0$. Similarly we have

$$
\overline{\mathcal{M}}+\mathcal{M}^{\dagger}=0
$$

if and only if $A=0$. As we have seen, these conditions are associated with (137) and (144) respectively.

The matrices $\Omega$ and $\Psi$ have the additional commutation properties

$$
\Psi \Omega^{\dagger}=\Omega \Psi, \quad \Omega^{\dagger} \Psi=\Psi \Omega
$$

which give

$$
\mathcal{M} \bar{M}=\left(A^{2}-B^{2}\right) \mathbf{I}
$$

and, along with (131)

$$
|\mathcal{M}|=D(1-D)=A^{2}-B^{2} .
$$

We also have

$$
\Psi \Omega+\bar{\Omega} \bar{\Psi}=\Psi \Omega-\Omega^{\dagger} \Psi=0
$$

and therefore

$$
\operatorname{Tr}(\Psi \Omega)=0 .
$$

We can now express the terms of $J_{x}$ and $J_{y}$ containing the matrix $\mathcal{M}$ in terms of the matrices $\Omega$ and $\Psi$ and the coefficients $A$ and $B$. Thus

$$
\begin{aligned}
& \widehat{\mathbf{X}}^{\dagger} \overline{\mathcal{M}} \widehat{\mathbf{Y}}=A\left\{\widehat{\mathbf{X}}^{\dagger} \Omega^{\dagger} \widehat{\mathbf{Y}}\right\}-B\left\{\hat{\mathbf{X}}^{\dagger} \Psi \widehat{\mathbf{Y}}\right\} \\
& \widehat{\mathbf{X}}^{\dagger} \mathcal{M}^{\dagger} \hat{\mathbf{Y}}=A\left\{\widehat{\mathbf{X}}^{\dagger} \Omega^{\dagger} \hat{\mathbf{Y}}\right\}+B\left\{\widehat{\mathbf{X}}^{\dagger} \Psi \widehat{\mathbf{Y}}\right\} \\
& \overline{\mathcal{M}}^{\dagger} \overline{\mathcal{M}}=\left(A^{2}+B^{2}\right) \mathbb{I}-2 A B \Omega \Psi \\
& \widehat{\mathbf{Y}}^{\dagger} \overline{\mathcal{M}}^{\dagger} \overline{\mathcal{M}} \widehat{\mathbf{Y}}=\left(A^{2}+B^{2}\right) c_{2} \\
& -2 A B\left\{\widehat{\mathbf{Y}}^{\dagger} \Omega \Psi \hat{\mathbf{Y}}\right\} \\
& \mathcal{M}^{\dagger} \mathcal{M}=\left(A^{2}+B^{2}\right) \mathbf{I}+2 A B \Psi \Omega \\
& \widehat{\mathrm{X}}^{\dagger} \mathcal{M}^{\dagger} \mathcal{M} \widehat{\mathrm{X}}=\left(A^{2}+B^{2}\right) \epsilon_{1} \\
& +2 A B\left\{\widehat{\mathbf{X}}^{\dagger} \Psi \Omega \hat{\mathbf{X}}\right\}
\end{aligned}
$$

and, using (163),

$$
\operatorname{Tr}\left(\mathcal{M} \mathcal{M}^{\dagger}\right)=\operatorname{Tr}\left(\mathcal{M}^{\dagger} \mathcal{M}\right)=2\left(A^{2}+B^{2}\right) .
$$

Using these results in (119), (120) and (108) we obtain

$$
\begin{aligned}
J_{x} & =F\left\{D^{2} \epsilon_{1}+(1-D)^{2} \epsilon_{2}\right\} \\
& +F\left\{\left(A^{2}+B^{2}\right)\left(\epsilon_{1}+\epsilon_{2}\right)\right\} \\
& +2 A F\left\{\widehat{\mathbf{X}}^{\dagger} \Omega^{\dagger} \hat{\mathbf{Y}}\right\} \\
& +2(1-2 D) B F\left\{\widehat{\mathbf{X}}^{\dagger} \Psi \widehat{\mathbf{Y}}\right\} \\
& +2 A B E\left\{\widehat{\mathbf{X}}^{\dagger} \Psi \Omega \widehat{\mathbf{X}}-\widehat{\mathbf{Y}}^{\dagger} \Omega \Psi \widehat{\mathbf{Y}}\right\}
\end{aligned}
$$

and

$$
\begin{aligned}
J_{y} & =F\left\{D^{2} \epsilon_{2}+(1-D)^{2} \epsilon_{1}\right\} \\
& +F\left\{\left(A^{2}+B^{2}\right)\left(\epsilon_{1}+\epsilon_{2}\right)\right\} \\
& -2 A F\left\{\widehat{\mathbf{X}}^{\dagger} \Omega^{\dagger} \widehat{\mathbf{Y}}\right\} \\
& +2(1-2 D) B F\left\{\widehat{\mathbf{X}}^{\dagger} \Psi \widehat{\mathbf{Y}}\right\} \\
& +2 A B F\left\{\widehat{\mathbf{X}}^{\dagger} \Psi \Omega \widehat{\mathbf{X}}-\widehat{\mathbf{Y}}^{\dagger} \Omega \Psi \widehat{\mathbf{Y}}\right\}
\end{aligned}
$$

where

$$
\begin{aligned}
F & =\left\{1-2 D(1-D)+2\left(A^{2}+B^{2}\right)\right\}^{-1 / 2} \\
& =\left\{1+4 B^{2}\right\}^{-1 / 2}
\end{aligned}
$$

To proceed further we compute

$$
\begin{aligned}
\widehat{\mathbf{X}}^{\dagger} \Omega^{\dagger} \widehat{\mathbf{Y}} & =\widehat{\mathbf{X}}_{0}^{\dagger}\left(\mathcal{A}^{n}\right)^{\dagger} \Omega^{\dagger} \mathcal{B}^{n} \widehat{\mathbf{Y}}_{0} \\
& =\widehat{\mathbf{X}}_{0}^{\dagger} \Omega^{\dagger}\left(\mathcal{A}^{n}\right)^{\dagger} \mathcal{B}^{n} \widehat{\mathbf{Y}}_{0} \\
\widehat{\mathbf{X}}^{\dagger} \Psi \widehat{\mathbf{Y}} & =\widehat{\mathbf{X}}_{0}^{\dagger}\left(\mathcal{A}^{n}\right)^{\dagger} \Psi \mathcal{B}^{n} \widehat{\mathbf{Y}}_{0} \\
& =\widehat{\mathbf{X}}_{0}^{\dagger} \Psi \mathcal{A}^{n} \mathcal{B}^{n} \widehat{\mathbf{Y}}_{0} \\
\widehat{\mathbf{Y}}^{\dagger} \Omega \Psi \widehat{\mathbf{Y}} & =\widehat{\mathbf{Y}}_{0}^{\dagger}\left(\mathcal{B}^{n}\right)^{\dagger} \Omega \Psi \mathcal{B}^{n} \widehat{\mathbf{Y}}_{0} \\
& =\widehat{\mathbf{Y}}_{0}^{\dagger} \Psi \Omega^{\dagger} \mathcal{B}^{2 n} \widehat{\mathbf{Y}}_{0} \\
\widehat{\mathbf{X}}^{\dagger} \Psi \Omega \hat{\mathbf{X}} & =\widehat{\mathbf{X}}_{0}^{\dagger}\left(\mathcal{A}^{n}\right)^{\dagger} \Psi \Omega \mathcal{A}^{n} \widehat{\mathbf{X}}_{0} \\
& =\widehat{\mathbf{X}}_{0}^{\dagger} \Psi \Omega \mathcal{A}^{2 n} \widehat{\mathbf{X}}_{0}
\end{aligned}
$$

where the components of $\widehat{\mathbf{X}}_{0}$ and $\widehat{\mathbf{Y}}_{0}$ are given by (70-71). We then have

$$
\begin{aligned}
& \widehat{\mathbf{X}}^{\dagger} \Omega^{\dagger} \hat{\mathbf{Y}}=\sqrt{\epsilon_{1} \epsilon_{2}} \cos \xi_{-} \\
& \widehat{\mathbf{X}}^{\dagger} \Psi \widehat{\mathbf{Y}}=\sqrt{\epsilon_{1} \epsilon_{2}} \cos \xi_{+}
\end{aligned}
$$




$$
\begin{aligned}
& \hat{\mathbf{Y}}^{\dagger} \Omega \Psi \widehat{\mathbf{Y}}=\epsilon_{2} \cos \zeta_{2} \\
& \widehat{\mathbf{X}}^{\dagger} \Psi \Omega \widehat{\mathbf{X}}=\epsilon_{1} \cos \zeta_{1}
\end{aligned}
$$

where

$$
\begin{aligned}
& \xi_{-}=n \psi_{1}-n \psi_{2}+\phi_{2}-\phi_{1}+\omega \\
& \xi_{+}=n \psi_{1}+n \psi_{2}-\phi_{1}-\phi_{2}+\psi \\
& \zeta_{2}=2 n \psi_{2}-2 \phi_{2}+\psi-\omega=\xi_{+}-\xi_{-} \\
& \zeta_{1}=2 n \psi_{1}-2 \phi_{1}+\psi+\omega=\xi_{+}+\xi_{-}
\end{aligned}
$$

and

$$
\psi_{1}=2 \pi Q_{1}, \quad \psi_{2}=2 \pi Q_{2} .
$$

Thus the horizontal and vertical amplitudes become

$$
\begin{aligned}
J_{x} & =F\left\{D^{2} \epsilon_{1}+(1-D)^{2} \epsilon_{2}\right\} \\
& +F\left\{\left(A^{2}+B^{2}\right)\left(\epsilon_{1}+\epsilon_{2}\right)\right\} \\
& +2 F \sqrt{\epsilon_{1} \epsilon_{2}}\left\{A \cos \xi_{-}\right\} \\
& +2 F \sqrt{\epsilon_{1} \epsilon_{2}}\left\{(1-2 D) B \cos \xi_{+}\right\} \\
& +2 A B F\left\{\epsilon_{1} \cos \zeta_{1}-\epsilon_{2} \cos \zeta_{2}\right\}
\end{aligned}
$$

and

$$
\begin{aligned}
J_{y} & =F\left\{D^{2} \epsilon_{2}+(1-D)^{2} \epsilon_{1}\right\} \\
& +F\left\{\left(A^{2}+B^{2}\right)\left(\epsilon_{1}+\epsilon_{2}\right)\right\} \\
& -2 F \sqrt{\epsilon_{1} \epsilon_{2}}\left\{A \cos \xi_{-}\right\} \\
& +2 F \sqrt{\epsilon_{1} \epsilon_{2}}\left\{(1-2 D) B \cos \xi_{+}\right\} \\
& +2 A B F\left\{\epsilon_{1} \cos \zeta_{1}-\epsilon_{2} \cos \zeta_{2}\right\}
\end{aligned}
$$

where

$$
D(1-D)=A^{2}-B^{2}
$$

and

$$
F^{\prime}=\left\{1+4 B^{2}\right\}^{-1 / 2} .
$$

These expressions are exact. They give the amplitudes of oscillation in the horizontal and vertical planes in terms of the initial condition parameters $\epsilon_{1}, \epsilon_{2}, \phi_{1}$ and $\phi_{2}$, and the parameters $D, A, B, \omega$, $w_{1}, Q_{1}$ and $Q_{2}$ of the one-turn matrix. The simple appearance of the parameters $A$ and $B$ in the expressions shows the utility of expressing the matrix $\mathcal{M}$ as $A \Omega+B \Psi$. Note that each amplitude contains frequencies $Q_{1}-Q_{2}, Q_{1}+Q_{2}, 2 Q_{1}$ and $2 Q_{2}$. If $B=0$, only frequency $Q_{1}-Q_{2}$ appears, while if $A=0$, only frequency $Q_{1}+Q_{2}$ appears. Note also that frequency $Q_{1}-Q_{2}$ is absent from the sum of the amplitudes and is the only frequency present in the difference.

\section{The Difference Resonance}

Let us examine further the case $B=0$. In this case we have $A^{2}=D(1-D)$ and $F=1$, and equations (187) and (188) become

$$
\begin{aligned}
J_{2} & =D \epsilon_{1}+(1-D) \epsilon_{2} \\
& +2\left\{D(1-D) \epsilon_{1} \epsilon_{2}\right\}^{1 / 2} \cos \xi_{-}
\end{aligned}
$$

and

$$
\begin{aligned}
J_{y} & =D \epsilon_{2}+(1-D) \epsilon_{1} \\
& -2\left\{D(1-D) \epsilon_{1} \epsilon_{2}\right\}^{1 / 2} \cos \xi_{-}
\end{aligned}
$$

where

$$
\xi_{-}=2 \pi n\left(Q_{1}-Q_{2}\right)+\phi_{2}-\phi_{1}+\omega .
$$

Here we see that the only $n$ dependence in the expressions for $J_{x}$ and $J_{y}$ is in the terms contain-

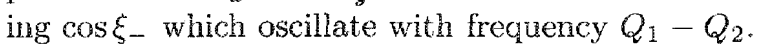
Moreover we have, as already shown,

$$
J_{x}+J_{y}=\epsilon_{1}+\epsilon_{2} .
$$

These are both characteristics of the amplitudes obtained in the standard hamiltonian treatment of the linear coupling difference resonance. We therefore identify the condition $B=0$ (or $B^{2} \ll A^{2}$ ) with the difference resonance. As we have seen, this condition is equivalent to $\overline{\mathcal{M}}=\mathcal{M}^{\dagger}$ which gives

$$
\mathcal{M}_{11}=\mathcal{M}_{22}, \quad \mathcal{M}_{12}=-\mathcal{M}_{21} \text {. }
$$

The work of Calaga, Tómas and Franchi [15] shows that the normalized coupling matrix elements of Sagan and Rubin [7] satisfy equations (195) when the sum resonance driving term of the hamiltonian treatment is zero. In Section 12 we shall see that $\mathcal{M}$ is in fact proportional to the normalized coupling matrix.

Since for $B=0$ we have

$$
D(1-D)=A^{2}-B^{2}=A^{2}>0
$$

it follows from (56) that we must have

$$
|\mathrm{m}+\overline{\mathbf{n}}|>0
$$

and we may define

$$
K^{2}=|\mathbf{m}+\overline{\mathbf{n}}| .
$$

Using this in (27) we then have

$$
U^{2}=T^{2}+4 K^{2}
$$


and (56) becomes

$$
D(1-D)=\frac{K^{2}}{T^{2}+4 K^{2}} .
$$

Substituting (200) into (191) and (192) we see that the oscillations of $J_{x}$ and $J_{y}$ are greatest when $T=$ 0 and go to zero as $K^{2}$ goes to zero. We also have

$$
D=\frac{1}{2}+\frac{1}{2}\left\{\frac{T^{2}}{T^{2}+4 K^{2}}\right\}^{1 / 2}
$$

which shows that $D$ goes to $1 / 2$ as $T$ goes to zero and goes to one as $K^{2}$ goes to zero. The parameters $T$ and $K$ correspond to the unperturbed tune separation and the coupling parameter in the standard treatment of the linear coupling resonance.

By specifying values for parameters $A, B, Q_{1}$, $Q_{2}$ we obtain values for $D, U^{2}, K^{2}$, and $T^{2}$. As an example of the difference resonance let us take

$$
\begin{aligned}
& A=0.48, \quad B=0.048 \\
& Q_{1}=5.2364, \quad Q_{2}=4.2236 .
\end{aligned}
$$

We then have

$$
\begin{aligned}
D & =0.6480 \\
U & =2 \cos \left(2 \pi Q_{1}\right)-2 \cos \left(2 \pi Q_{2}\right) \\
& =-0.1595 \\
K^{2} & =D(1-D) U^{2}=0.005806
\end{aligned}
$$

and

$$
T^{2}=U^{2}-4 K^{2}=0.002230 .
$$

Figure 1 shows a plot of $J_{x}, J_{y}$ and $J_{x}+J_{y}$ obtained with these values. Here we have taken $\epsilon_{1}=\epsilon_{2}=1$, $\phi_{1}=\phi_{2}=0$ and $\omega=\psi=\pi / 4$. As expected we see $J_{x}$ and $J_{y}$ oscillations characteristic of the difference resonance. The small-amplitude highfrequency oscillations seen on all of the curves are due to the parameter $B$ being small but nonzero. If we set $B=0$ and keep $A, Q_{1}, Q_{2}$ the same as before, we obtain the curves shown in Figure 2. Here we see that the high-frequency oscillations are gone and the sum $J_{x}+J_{y}$ is constant. Setting $B=0$ corresponds to discarding the high-frequency terms in the hamiltonian treatment of the difference resonance.

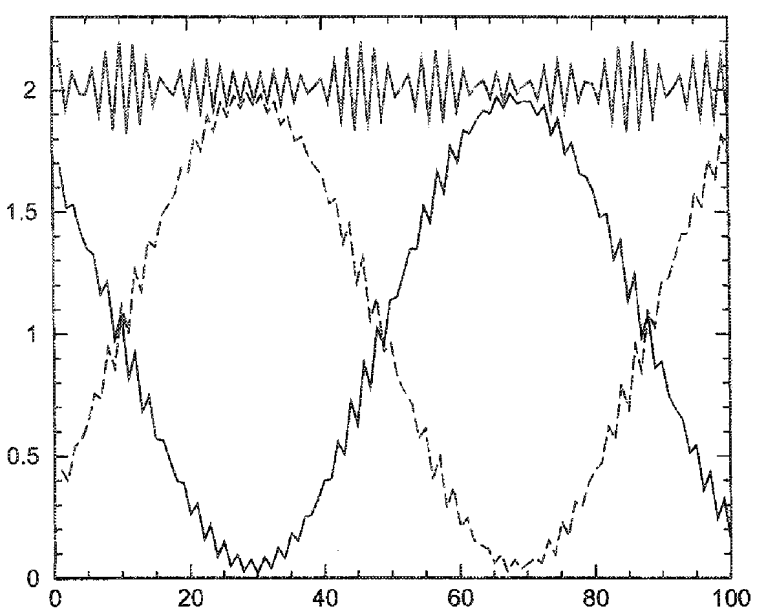

Figure 1: Horizontal and vertical amplitudes $J_{x}$ and $J_{y j}$ and their sum versus turn plotted in black, red (dashod curvo) and blue respectively. Here normalized coupling matrix parameters $A=0.48$ and $B=0.048$. The small-amplitude high-frequency oscillations seen on all of the curves are due to the parametor $B$ being small but nonzero.

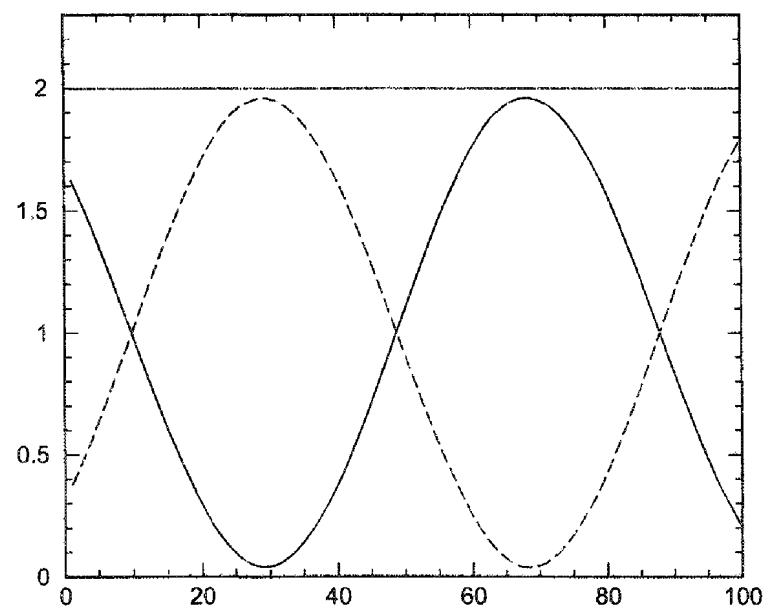

Figure 2: Amplitudes $J_{x}$ and $J_{y}$ and their sum obtained with normalized coupling matrix parameters $A=0.48$ and $B=0$. Here the high-frequency oscillations are gone and the sum is constant. 


\section{The Sum Resonance}

For the case $A=0$ we have $B^{2}=D(D-1)$, $F=\{2 D-1\}^{-1}$ and equations (187) and (188) become

$$
\begin{aligned}
J_{x} & =D \epsilon_{1}+(D-1) \epsilon_{2} \\
& -2\left\{D(D-1) \epsilon_{1} \epsilon_{2}\right\}^{1 / 2} \cos \xi_{+}
\end{aligned}
$$

and

$$
\begin{aligned}
J_{y} & =D \epsilon_{2}+(D-1) \epsilon_{1} \\
& -2\left\{D(D-1) \epsilon_{1} \epsilon_{2}\right\}^{1 / 2} \cos \xi_{+}
\end{aligned}
$$

where

$$
\xi_{+}=2 \pi n\left(Q_{1}+Q_{2}\right)-\phi_{1}-\phi_{2}+\psi
$$

Here we see that the only $n$ dependence in the expressions for $J_{x}$ and $J_{y}$ is in the terms containing $\cos \xi_{+}$which oscillate with frequency $Q_{1}+Q_{2}$. Moreover we have

$$
J_{x}-J_{y}=\epsilon_{1}-\epsilon_{2} .
$$

These are just the characteristics of the amplitudes obtained in the standard hamiltonian treatment of the linear coupling sum resonance. We therefore identify the condition $A=0$ (or $A^{2} \ll B^{2}$ ) with the sum resonance. Note that this condition is equivalent to $\overline{\mathcal{M}}=-\mathcal{M}^{\dagger}$ which gives

$$
\mathcal{M}_{11}=-\mathcal{M}_{22}, \quad \mathcal{M}_{12}=\mathcal{M}_{21} .
$$

The work of Ref. [15] shows that the normalized coupling matrix elements satisfy these equations when the difference resonance driving term (of the hamiltonian treatment) is zero.

Since for $A=0$ we have

$$
D(D-1)=B^{2}-A^{2}=B^{2}>0
$$

it follows from (56) that we must have

$$
|\mathrm{m}+\overline{\mathbf{n}}|<0 .
$$

Thus we may define

$$
K^{2}=-|\mathbf{m}+\tilde{\mathbf{n}}|
$$

and we have

$$
D(D-1)=\frac{K^{2}}{T^{2}-4 K^{2}} .
$$

Substituting (216) into (208) and (209) we see that in this case the oscillations of $J_{x}$ and $J_{y}$ increase

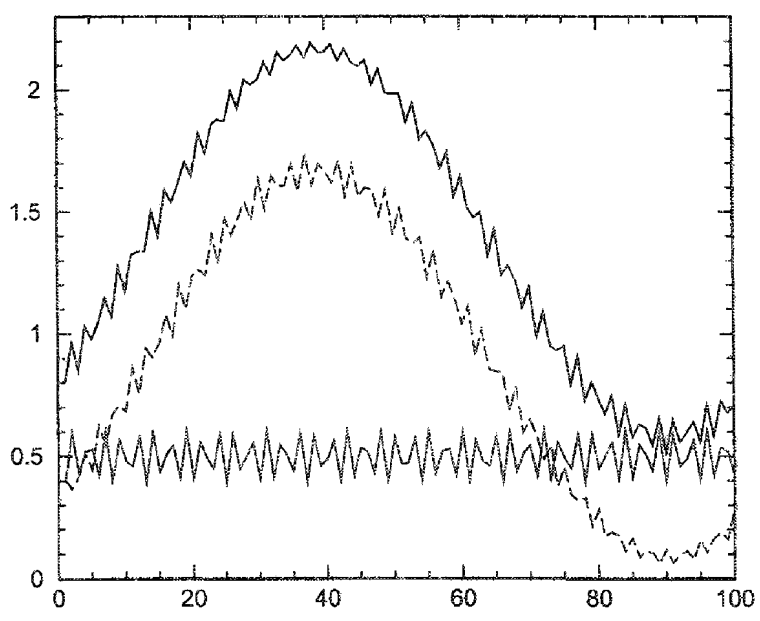

Figure 3: Amplitudes $J_{x}$ and $J_{y}$ and their difference plotted in black, red (dashed curve) and blue respectively. Here normalized coupling matrix parameters $A=0.056$ and $B=$ 0.56. The small-amplitude high-frequency oscillations seen on all of the curves are due to the parameter $A$ being small but nonzero.

without bound as $T^{2}$ approaches $4 K^{2}$. The motion is unstable for $T^{2} \leq 4 K^{2}$. We also have

$$
D=\frac{1}{2}+\frac{1}{2}\left\{\frac{T^{2}}{T^{2}-4 K^{2}}\right\}^{1 / 2}
$$

which shows that $D$ becomes large as $T^{2}$ approaches $4 K^{2}$ and goes to one as $K^{2}$ goes to zero. As an example of the sum resonance let us take

$$
\begin{aligned}
& A=0.056, \quad B=0.56 \\
& Q_{1}=5.2124, \quad Q_{2}=4.7973 .
\end{aligned}
$$

We then we have

$$
\begin{aligned}
D & =1.2486 \\
U & =2 \cos \left(2 \pi Q_{1}\right)-2 \cos \left(2 \pi Q_{2}\right) \\
& =-0.1176 \\
K^{2} & =D(D-1) U^{2}=0.004291
\end{aligned}
$$

and

$$
T^{2}=U^{2}+4 K^{2}=0.030987
$$

Figure 3 shows a plot of $J_{x}, J_{y}$ and $J_{x}-J_{y}$ obtained with these values. Here we have taken $\epsilon_{1}=1, \epsilon_{2}=0.5, \phi_{1}=\phi_{2}=0$ and $\omega=\psi=\pi / 4$. As expected we see $J_{x}$ and $J_{y}$ oscillations characteristic of the sum resonance. The small-amplitude highfrequency oscillations seen on all of the curves are due to the parameter $A$ being small but nonzero. 


\section{Change of Representation}

The matrix $\mathcal{W}$ in the normal-form expression (32) for $\mathrm{T}$ is not unique. In the Appendix it is show that if there is another symplectic matrix $\mathcal{W}$ for which

$$
\mathrm{T}=\underline{W U}^{-1}
$$

then we have

$$
\underline{W}=\text { WO }
$$

where $O$ must be of the form

$$
\mathcal{O}=\left(\begin{array}{ll}
\mathrm{P} & 0 \\
0 & \mathrm{Q}
\end{array}\right)
$$

with

$$
P=\left(\begin{array}{rr}
\cos \omega_{1} & \sin \omega_{1} \\
-\sin \omega_{1} & \cos \omega_{1}
\end{array}\right)
$$

and

$$
\mathbf{Q}=\left(\begin{array}{cc}
\cos \omega_{2} & \sin \omega_{2} \\
-\sin \omega_{2} & \cos \omega_{2}
\end{array}\right)
$$

We shall call the transformation from $\mathcal{W}$ to $\underline{\mathcal{W}}$ a change in the normal-form representation of $\mathbf{T}$. The transformation is generated by the matrix $\mathcal{O}$ and we use an underline to denote a transformed parameter or matrix. The purpose here is to identify which parameters and matrices of the previous sections are independent of the representation.

It follows from (225-228) that

$$
\underline{\mathcal{W}} \underline{\mathcal{W}}^{\dagger}=\mathcal{W} \mathcal{O} \mathcal{O}^{\dagger} \mathcal{W}^{\dagger}=\mathcal{W} \mathcal{W}^{\dagger}
$$

and therefore

$$
\underline{\mathbf{E}}=\underline{\mathcal{W}} \underline{\mathcal{W}}^{\dagger}=\mathcal{W} \mathcal{W}^{\dagger}=\mathbf{E} .
$$

Thus $\mathbf{E}$ is unchanged by a change of representation. The same is obviously true for the submatrices $(\mathbf{F}$, $\mathbf{G}$ and $\mathbf{C}$ ) of $\mathbf{E}$ and it follows from (92) and (93) that the amplitudes $J_{x}$ and $J_{y}$ are unchanged.

Writing

$$
\begin{aligned}
\underline{W} & =\left(\begin{array}{ll}
\underline{\mathcal{W}}_{1} & \underline{\mathcal{D}}_{1} \\
\underline{\mathcal{D}}_{2} & \underline{\mathcal{W}}_{2}
\end{array}\right) \\
& =\left(\begin{array}{ll}
\mathcal{W}_{1} & \mathcal{D}_{1} \\
\mathcal{D}_{2} & \mathcal{W}_{2}
\end{array}\right)\left(\begin{array}{ll}
\mathbf{P} & 0 \\
\mathbf{0} & \mathbf{Q}
\end{array}\right)
\end{aligned}
$$

we have

$$
\begin{aligned}
& \underline{W}_{1}=\mathcal{W}_{1} \mathbf{P}, \quad \underline{D}_{1}=\mathcal{D}_{1} \mathbf{Q} \\
& \underline{\mathcal{D}}_{2}=\mathcal{D}_{2} \mathbf{P}, \quad \underline{\mathcal{W}}_{2}=\mathcal{W}_{2} \mathbf{Q} .
\end{aligned}
$$

Since $\mathbf{P} \mathbf{P}^{\dagger}=\mathbf{Q} \mathbf{Q}^{\dagger}=\mathbf{I}$ it follows that

$$
\begin{aligned}
& \underline{\mathcal{W}}_{1} \underline{\mathcal{W}}_{1}^{\dagger}=\mathcal{W}_{1} \mathcal{W}_{1}^{\dagger} \\
& \underline{\mathcal{D}}_{1} \mathcal{D}_{1}^{\dagger}=\mathcal{D}_{1} \mathcal{D}_{1}^{\dagger} \\
& \underline{\mathcal{W}}_{2} \underline{\mathcal{W}}_{2}^{\dagger}=\mathcal{W}_{2} \mathcal{W}_{2}^{\dagger} \\
& \underline{\mathcal{D}}_{2} \underline{\mathcal{D}}_{2}^{\dagger}=\mathcal{D}_{2} \mathcal{D}_{2}^{\dagger}
\end{aligned}
$$

which shows that these symmetric positivedefinite matrices are unchanged by a change in representa. tion. We also have

$$
\begin{aligned}
& \left|\underline{\mathcal{W}}_{1}\right|=\left|\mathcal{W}_{1}\right|=D \\
& \left|\underline{\mathcal{D}}_{1}\right|=\left|\mathcal{D}_{1}\right|=1-D \\
& \left|\underline{\mathcal{W}}_{2}\right|=\left|\mathcal{W}_{2}\right|=D \\
& \left|\underline{\mathcal{D}}_{2}\right|=\left|\mathcal{D}_{2}\right|=1-D
\end{aligned}
$$

which shows that the parameter $D$ is unchanged. This is also evident from inspection of equation (56).

For the matrix $\mathcal{M}$ we have

$$
\begin{aligned}
& \underline{\mathcal{M}}=\overline{\mathcal{D}}_{1} \mathcal{W}_{\mathbf{1}}=\overline{\mathbf{Q}}_{\overline{\mathcal{D}}_{1}} \mathcal{W}_{1} \mathbf{P}=\overline{\mathbf{Q}} \mathcal{M} \mathbf{P} \\
& \underline{\mathcal{M}}=A \overline{\mathbf{Q}} \Omega \mathbf{P}+B \overline{\mathbf{Q}} \Psi \mathbf{P}
\end{aligned}
$$

and

$$
\underline{\mathcal{M}}=A \underline{\Omega}+B \underline{\Psi}
$$

where

$$
\begin{aligned}
& \underline{\Omega}=\overline{\mathbf{Q}} \Omega \mathbf{P}=\left(\begin{array}{rr}
\cos \underline{\omega} & \sin \underline{\omega} \\
-\sin \underline{\omega} & \cos \underline{\omega}
\end{array}\right) \\
& \underline{\Psi}=\overline{\mathbf{Q}} \Psi \mathbf{P}=\left(\begin{array}{cc}
\cos \underline{\psi} & \sin \psi \\
\sin \underline{\psi} & -\cos \underline{\psi}
\end{array}\right)
\end{aligned}
$$

and

$$
\begin{aligned}
& \underline{\omega}=\omega+\omega_{1}-\omega_{2} \\
& \underline{\psi}=\psi+\omega_{1}+\omega_{2} .
\end{aligned}
$$

Thus $\mathcal{M}$ does in fact change under a change in representation, but only the phases $\omega$ and $\psi$ change; the parameters $A$ and $B$ are unchanged.

There is a corresponding change in the phases $\phi_{1}$ and $\phi_{2}$ associated with the normalized coordinates. The transformed normalized coordinates are given by

$$
\underline{\underline{Z}}_{0}=\underline{\mathcal{W}}^{-1} \mathbf{Z}_{0}
$$


where

$$
\begin{aligned}
& \underline{\underline{\mathbf{z}}}_{0}=\left(\begin{array}{l}
\widehat{\widehat{\mathbf{X}}}_{0} \\
\underline{\widehat{\mathbf{Y}}}_{0}
\end{array}\right) \\
& \underline{\hat{\mathbf{x}}}_{0}=\left(\begin{array}{c}
\widehat{\underline{X}}_{0} \\
\widehat{\underline{X}}_{0}^{\prime}
\end{array}\right), \quad \hat{\underline{Y}}_{0}=\left(\begin{array}{c}
\hat{\underline{Y}}_{0} \\
\widehat{\widehat{Y}}_{0}^{\prime}
\end{array}\right) .
\end{aligned}
$$

Using (225) and (59) we have

$$
\begin{aligned}
& \underline{\mathbf{Z}}_{0}=\mathcal{O}^{-1} \mathcal{W}^{-1} \mathbf{Z}_{0}=\mathcal{O}^{-1} \hat{\mathbf{Z}}_{0} \\
& \underline{\mathbf{X}}_{0}=\mathbf{P}^{\dagger} \widehat{\mathbf{X}}_{0} . \quad \widehat{\mathbf{Y}}_{0}=\mathbf{Q}^{\dagger} \hat{\mathbf{Y}}_{0}
\end{aligned}
$$

Thus we have

$$
\begin{aligned}
& \underline{\mathbf{\mathbf { X }}}_{0}^{\dagger} \widehat{\hat{\mathbf{X}}}_{0}=\hat{\mathbf{X}}_{0}^{\dagger} \widehat{\mathbf{X}}_{0}=\epsilon_{1} \\
& \underline{\hat{\mathbf{Y}}}_{0}^{\dagger} \underline{\hat{\mathbf{Y}}}_{0}=\widehat{\mathbf{Y}}_{0}^{\dagger} \widehat{\mathbf{Y}}_{0}=\epsilon_{2}
\end{aligned}
$$

and

$$
\begin{array}{ll}
\hat{X}_{0}=\sqrt{\epsilon_{1}} \cos \underline{\phi}_{1}, & \underline{X}_{0}^{\prime}=\sqrt{\epsilon_{1}} \sin \underline{\phi}_{1} \\
\hat{\underline{Y}}_{0}=\sqrt{\underline{\epsilon}_{2}} \cos \underline{\phi}_{2}, & \underline{\hat{Y}}_{0}^{\prime}=\sqrt{\underline{\epsilon}_{2}} \sin \underline{\phi}_{2}
\end{array}
$$

where

$$
\begin{aligned}
& \phi_{1}=\phi_{1}+\omega_{1} \\
& \phi_{2}=\phi_{2}+\omega_{2} .
\end{aligned}
$$

The change in representation therefore shifts phases $\phi_{1}$ and $\phi_{2}$ by $\omega_{1}$ and $\omega_{2}$ respectively. Note that these equations along with (247) and (248) give

$$
\begin{aligned}
& \underline{\phi}_{2}-\underline{\phi}_{1}+\underline{\omega}=\phi_{2}-\phi_{1}+\omega \\
& -\underline{\phi}_{1}-\underline{\phi}_{2}+\underline{\psi}=-\phi_{1}-\phi_{2}+\psi
\end{aligned}
$$

which shows that the phases $\xi_{+}, \xi_{-}, \zeta_{1}$ and $\zeta_{2}$ appearing in the expressions for $J_{x}$ and $J_{y}$ are unchanged by a change in representation.

\section{The Edwards-Teng Parameters}

In terms of the matrix $\mathcal{M}$ we have

$$
\mathcal{D}_{1}=\frac{1}{D} \mathcal{W}_{1} \overline{\mathcal{M}}, \quad \mathcal{D}_{2}=-\frac{1}{D} \mathcal{W}_{2} \mathcal{M}
$$

and

$$
\mathcal{W}=\frac{1}{D}\left(\begin{array}{cc}
D \mathcal{W}_{1} & \mathcal{W}_{1} \overline{\mathcal{M}} \\
-\mathcal{W}_{2} \mathcal{M} & D \mathcal{W}_{2}
\end{array}\right)
$$

which we can write as

$$
\mathcal{W}=\frac{1}{D} \mathcal{R}\left(\begin{array}{cc}
\mathcal{W}_{1} & 0 \\
0 & \mathcal{W}_{2}
\end{array}\right)
$$

where

$$
\mathcal{R}=\left(\begin{array}{cc}
D \mathrm{I} & \mathcal{W}_{1} \overline{\mathcal{M}} \mathcal{W}_{2}^{-1} \\
-\mathcal{W}_{2} \mathcal{M} W_{1}^{-1} & D \mathbb{I}
\end{array}\right)
$$

Defining

$$
d=\sqrt{D} . \quad \widehat{\mathcal{F}}=\frac{1}{d} \mathcal{W}_{1}, \quad \widehat{\mathcal{G}}=\frac{1}{d} \mathcal{W}_{2}
$$

and

$$
\mathbf{W}=\frac{1}{d} W_{2} \mathcal{M} W_{1}^{-1}=\frac{1}{d} \widehat{\mathcal{G}} M \widehat{\mathcal{F}}^{-1}
$$

we then have

$$
\mathcal{W}=\mathbf{R} \hat{N}
$$

where

$$
\begin{aligned}
& \mathbf{R}=\left(\begin{array}{cc}
d \mathbf{I} & \overline{\mathbf{W}} \\
-\mathbf{W} & d \mathbf{I}
\end{array}\right) \\
& \hat{\mathcal{N}}=\left(\begin{array}{cc}
\widehat{\mathcal{F}} & \mathbf{0} \\
\mathbf{0} & \widehat{\mathcal{G}}
\end{array}\right) .
\end{aligned}
$$

The one-turn matrix is then

$$
\mathbf{T}=\mathcal{W} \mathcal{W} \mathcal{W}^{-1}=\mathbf{R U R}^{-1}
$$

where

$$
\mathbf{U}=\widehat{\mathcal{N}} \mathcal{U} \widehat{\mathcal{N}}^{-1} .
$$

We shall show that this is just the parameterization of Edwards and Teng $[4,5,6]$. Furthermore we show that $\mathcal{M}$ is proportional to the normalized coupling matrix introduced by Sagan and Rubin [7].

Since $\hat{\mathcal{F}} \hat{\mathcal{F}}^{\dagger}$ and $\hat{\mathcal{G}} \widehat{\mathcal{G}}^{\dagger}$ are symmetric positivedefinite matrices with unit determinant we may define Courant-Snyder parameters $\alpha_{1}, \beta_{1}, \gamma_{1}, \alpha_{2}, \beta_{2}$ and $\gamma_{2}$ such that

$$
\left(\begin{array}{rr}
\beta_{1} & -\alpha_{\bar{I}} \\
-\alpha_{1} & \gamma_{1}
\end{array}\right)=\widehat{\mathcal{F}} \hat{\mathcal{F}}^{\dagger}=\frac{1}{D} \mathcal{W}_{1} \mathcal{W}_{1}^{\dagger}
$$

and

$$
\left(\begin{array}{rr}
\beta_{2} & -\alpha_{2} \\
-\alpha_{2} & \gamma_{2}
\end{array}\right)=\widehat{\mathcal{G}} \widehat{\mathcal{G}}^{\dagger}=\frac{1}{D} \mathcal{W}_{2} \mathcal{W}_{2}^{\dagger} .
$$

Equations (234) and (236) show that these parameters are unchanged by a change in representation. Using (232), (233) and (242) we also have

$$
\underline{W}_{2} \underline{\mathcal{M}} \underline{\mathcal{W}}_{1}^{-1}=\mathcal{W}_{2} \mathcal{M} \mathcal{W}_{1}^{-1}
$$


which shows that $\mathbf{W}$ and $\mathbf{R}$ are unchanged by a change in representation.

Defining now

$$
\begin{aligned}
& \mathcal{F}=\frac{1}{\sqrt{\beta_{1}}}\left(\begin{array}{rr}
\beta_{1} & 0 \\
-\alpha_{1} & 1
\end{array}\right) \\
& \mathcal{G}=\frac{1}{\sqrt{\beta_{2}}}\left(\begin{array}{rr}
\beta_{2} & 0 \\
-\alpha_{2} & 1
\end{array}\right)
\end{aligned}
$$

we have

$$
\begin{aligned}
& \mathcal{F} \mathcal{F}^{\dagger}=\left(\begin{array}{rr}
\beta_{1} & -\alpha_{1} \\
-\alpha_{1} & \gamma_{1}
\end{array}\right)=\hat{\mathcal{F}} \hat{\mathcal{F}} \\
& \mathcal{G} \mathcal{G}^{\dagger}=\left(\begin{array}{rr}
\beta_{2} & -\alpha_{2} \\
-\alpha_{2} & \gamma_{2}
\end{array}\right)=\widehat{\widehat{G}} \hat{G}^{\dagger}
\end{aligned}
$$

and it follows that we must have

$$
\hat{\mathcal{F}}=\mathcal{F} \mathbf{P}, \quad \widehat{\mathcal{G}}=\mathcal{G Q}
$$

where

$$
\begin{aligned}
& \mathbf{P}=\left(\begin{array}{rr}
C_{1} & S_{1} \\
-S_{1} & C_{1}
\end{array}\right) \\
& \mathbf{Q}=\left(\begin{array}{rr}
C_{2} & S_{2} \\
-S_{2} & C_{2}
\end{array}\right)
\end{aligned}
$$

and

$$
C_{1}^{2}+S_{1}^{2}=1, \quad C_{2}^{2}+S_{2}^{2}=1 .
$$

Thus we have

$$
\widehat{\mathcal{N}}=\mathcal{N O}
$$

where

$$
\mathcal{N}=\left(\begin{array}{cc}
\mathcal{F} & 0 \\
0 & \mathcal{G}
\end{array}\right), \quad \mathcal{O}=\left(\begin{array}{cc}
\mathbf{P} & \mathbf{0} \\
\mathbf{0} & \mathbf{Q}
\end{array}\right)
$$

and therefore

$$
\mathrm{U}=\mathcal{N O U O}^{-1} \mathcal{N}^{-1}=\mathcal{N} \mathcal{U N}^{-1}
$$

Carrying out the matrix multiplications we then find

$$
\mathbf{U}=\left(\begin{array}{cc}
\mathbf{A} & 0 \\
0 & \mathbf{B}
\end{array}\right)
$$

where

$$
\mathrm{A}=\mathcal{F} \mathcal{A F}^{-1}, \quad \mathrm{~B}=\mathcal{G B \mathcal { G } ^ { - 1 }}
$$

This along with the matrix $\mathbf{R}$ gives the parameterization of Edwards and Teng.
Note that we may always choose the representation such that

$$
\hat{\mathcal{F}}=\mathcal{F}, \quad \hat{\mathcal{G}}=\mathcal{G} .
$$

Equation (267) then becomes

$$
W=\frac{1}{d} \mathcal{G} M F^{-1}
$$

and we have

$$
M=d w
$$

where

$$
W=\mathcal{G}^{-1} \mathrm{WF}
$$

This is just the normalized coupling matrix introduced by Sagan and Rubin [7]. We also have, with the help of (262) and (266),

$$
\begin{array}{ll}
\mathcal{W}_{1}=d \mathcal{F}, & \mathcal{D}_{1}=\mathcal{F} \overline{\mathrm{W}} \\
\mathcal{D}_{2}=-\mathcal{G} \mathbf{w}, & \mathcal{W}_{2}=d \mathcal{G} .
\end{array}
$$

Equations (75-76) then become

$$
\begin{aligned}
& \mathbf{X}=d \mathcal{F} \mathcal{A}^{n} \widehat{\mathbf{X}}_{0}+\mathcal{F} \mathbf{w} \mathcal{B}^{n} \widehat{\mathbf{Y}}_{0} \\
& \mathbf{Y}=-\mathcal{G} \mathbf{w} \mathcal{A}^{n} \widehat{\mathbf{X}}_{0}+d \mathcal{G B} \mathcal{B}^{n} \widehat{\mathbf{Y}}_{0}
\end{aligned}
$$

which give the turn-by-turn positions and angles in terms of the Edwards-Teng parameters and the initial normalized coordinates.

Note that using (150) in (291) we have

$$
\mathbf{w}=\frac{1}{d}\{A \Omega+B \Psi\}
$$

where $\Omega$ and $\Psi$ are given by (151) and (152), and $d$ is given by

$$
d^{2}\left(1-d^{2}\right)=A^{2}-B^{2}
$$

The phases $\omega$ and $\psi$ in the matrices $\Omega$ and $\Psi$ are fixed by the choice (289). Thus, the one-turn matrix $\mathbf{T}$ is specified by the ten parameters $Q_{1}, Q_{2}$, $\alpha_{1}, \alpha_{2}, \beta_{1}, \beta_{2} A, B, \omega$ and $\psi$.

\section{Measurement of Coupling Parameters for the Case of the Difference Resonance}

If we are operating under the difference resonance condition $B=0$, then the number of parameters needed to specify the one-turn matrix is reduced from ten to eight. Measurements of the turn-byturn horizontal and vertical positions at a single 
dual-plane beam position monitor (BPM) then become sufficient to determine the coupling strength $K^{2}=|m+\bar{n}|$ and the phase $\omega$. To show this, we have for the case $B=0$,

$$
w=\sqrt{1-d^{2}} \Omega
$$

and equations (295) and (296) become

$$
\mathcal{F}^{-1} \mathrm{X}=d \mathcal{A}^{n} \widehat{\mathrm{X}}_{0}+e \Omega^{\dagger} B^{n} \widehat{\mathrm{Y}}_{0}
$$

and

$$
\mathfrak{G}^{-1} \mathrm{Y}=-e \Omega \mathcal{A}^{n} \widehat{\mathrm{X}}_{0}+d \mathcal{B}^{n} \widehat{\mathrm{Y}}_{0}
$$

where

$$
e=\sqrt{1-d^{2}}
$$

Using the identity

$$
\begin{array}{r}
\left(\begin{array}{rr}
\cos \theta & \sin \theta \\
-\sin \theta & \cos \theta
\end{array}\right)\left(\begin{array}{c}
\cos \chi \\
\sin \chi
\end{array}\right)= \\
\left(\begin{array}{r}
\cos (\theta-\chi) \\
-\sin (\theta-\chi)
\end{array}\right)
\end{array}
$$

we then have

$$
\begin{array}{r}
\mathcal{F}^{-1} \mathbf{X}=\sqrt{\epsilon_{1} d^{2}}\left(\begin{array}{r}
\cos \left(n \psi_{1}-\phi_{1}\right) \\
-\sin \left(n \psi_{1}-\phi_{1}\right)
\end{array}\right) \\
+\sqrt{\epsilon_{2} e^{2}}\left(\begin{array}{r}
\cos \left(n \psi_{2}-\phi_{2}-\omega\right) \\
-\sin \left(n \psi_{2}-\phi_{2}-\omega\right)
\end{array}\right)
\end{array}
$$

and

$$
\begin{array}{r}
\mathcal{G}^{-1} \mathbf{Y}=\sqrt{\epsilon_{2} d^{2}}\left(\begin{array}{r}
\cos \left(n \psi_{2}-\phi_{2}\right) \\
-\sin \left(n \psi_{2}-\phi_{2}\right)
\end{array}\right) \\
-\sqrt{\epsilon_{1} e^{2}}\left(\begin{array}{r}
\cos \left(n \psi_{1}-\phi_{1}+\omega\right) \\
-\sin \left(n \psi_{1}-\phi_{1}+\omega\right)
\end{array}\right)
\end{array}
$$

which gives turn-by-turn positions

$$
\begin{aligned}
X & =A_{1} \cos \left(n \psi_{1}+\chi_{1}\right) \\
& +A_{2} \cos \left(n \psi_{2}+\chi_{2}\right) \\
Y & =B_{1} \cos \left(n \psi_{1}+\eta_{1}\right) \\
& +B_{2} \cos \left(n \psi_{2}+\eta_{2}\right)
\end{aligned}
$$

where

$$
\begin{aligned}
& A_{1}=\sqrt{\epsilon_{1} \beta_{1} d^{2}}, \quad A_{2}=\sqrt{\epsilon_{2} \beta_{1} e^{2}} \\
& B_{1}=\sqrt{\epsilon_{1} \beta_{2} e^{2}}, \quad B_{2}=\sqrt{\epsilon_{2} \beta_{2} d^{2}} \\
& \chi_{1}=-\phi_{1}, \quad \chi_{2}=-\phi_{2}-\omega \\
& \eta_{1}=\pi-\phi_{1}+\omega, \quad \eta_{2}=-\phi_{2}
\end{aligned}
$$

and

$$
\psi_{1}=2 \pi Q_{1}, \quad \psi_{2}=2 \pi Q_{2}
$$

We can fit (306) and (307) to turn-by-turn measurements of the horizontal and vertical positions. Using the fited values of parameters $A_{1}, A_{2}, B_{1}$ and $B_{2}$ we obtain

$$
d^{2} e^{2} \epsilon_{1} \beta_{1} \epsilon_{2} B_{2}=A_{1} A_{2} B_{1} B_{2}
$$

and

$$
\sqrt{\epsilon_{1} \beta_{1} \epsilon_{2} \beta_{2}}=A_{1} B_{2}+A_{2} B_{1}
$$

which gives

$$
d^{2}\left(1-d^{2}\right)=\frac{A_{1} A_{2} B_{1} B_{2}}{\left(A_{1} B_{2}+A_{2} B_{1}\right)^{2}}
$$

Note that this expression is independent of which normal mode is labeled 1 and which is labeled 2 . The expression is not valid if $\epsilon_{1}=0$ or $\epsilon_{2}=0$, but one can always kick the beam so that both normal modes are excited.

Using the fitted values of $Q_{1}$ and $Q_{2}$ we also have

$$
U=2 \cos \left(2 \pi Q_{1}\right)-2 \cos \left(2 \pi Q_{2}\right)
$$

We then can calculate the coupling strength

$$
K^{2}=d^{2}\left(1-d^{2}\right) U^{2}
$$

and also

$$
T^{2}=U^{2}-4 K^{2}
$$

From the fitted parameters $\chi_{2}$ and $\eta_{2}$ we obtain the coupling phase

$$
\omega=\eta_{2}-\chi_{2}
$$

We also have

$$
\eta_{1}-\chi_{1}=\eta_{2}-\chi_{2}+\pi
$$

Which shows that $\omega$ is determined only to within a phase of $\pi$ due to the freedom to choose which normal mode is labeled 1 and which is labeled 2. Luo has obtained these results with his matrix perturbation approach [16] and with the standard hamiltonian approach [17]. 


\section{The Ripken Parameters}

For completeness we show here that the normalform matix $W$ provides a straight-forward route to the coupled lattice parameters introduced by Ripken $[8,9]$. Since $\mathcal{W}_{1} \mathcal{W}_{1}^{\dagger}, \mathcal{D}_{1} \mathcal{D}_{1}^{\dagger}, \mathcal{W}_{2} \mathcal{W}_{2}^{\dagger}$ and $\mathcal{D}_{2} \mathcal{D}_{2}^{\dagger}$ are symmetric positive-definite matrices with determinants

$$
\begin{aligned}
& \left|W_{1} W_{1}^{\dagger}\right|=\left|W_{2} W_{2}^{\dagger}\right|=D^{2} \\
& \left|\mathcal{D}_{1} \mathcal{D}_{1}^{\dagger}\right|=\left|\mathcal{D}_{2} \mathcal{D}_{2}^{\dagger}\right|=(1-D)^{2}
\end{aligned}
$$

we can introduce Courant-Snyder parameters defined by

$$
\begin{array}{ll}
D F_{1}=W_{1} W_{1}^{\dagger}, & \quad 1-D \mid F_{1}=\mathcal{D}_{1} \mathcal{D}_{1}^{\dagger} \\
D E_{2}=W_{2} W_{2}^{\dagger}, & 1-D \mid F_{2}=\mathcal{D}_{2} \mathcal{D}_{2}^{\dagger}
\end{array}
$$

where

$$
\begin{aligned}
& \mathbf{E}_{1}=\left(\begin{array}{rr}
\beta_{1} & -\alpha_{1} \\
-\alpha_{1} & \gamma_{1}
\end{array}\right) \\
& \mathbf{F}_{1}=\left(\begin{array}{rr}
b_{1} & -a_{1} \\
-a_{1} & g_{1}
\end{array}\right) \\
& \mathbf{E}_{2}=\left(\begin{array}{rr}
\beta_{2} & -\alpha_{2} \\
-\alpha_{2} & \gamma_{2}
\end{array}\right) \\
& \mathbf{F}_{2}=\left(\begin{array}{rr}
b_{2} & -a_{2} \\
-a_{2} & g_{2}
\end{array}\right) .
\end{aligned}
$$

It follows from equations (234-237) and (238-241) that these parameters are unchanged by a change in representation.

From equations (73-74) we see that the turn-byturn motion in the horizontal and vertical planes is given by

$$
\begin{aligned}
& \mathbf{X}=\mathbf{X}_{1}+\mathbf{X}_{2} \\
& \mathbf{Y}=\mathbf{Y}_{1}+\mathbf{Y}_{2}
\end{aligned}
$$

where

$$
\begin{array}{ll}
\mathbf{X}_{1}=\mathcal{W}_{1} \widehat{\mathbf{X}}, & \mathbf{X}_{2}=\mathcal{D}_{1} \widehat{\mathbf{Y}} \\
\mathbf{Y}_{1}=\mathcal{D}_{2} \widehat{\mathbf{X}}, & \mathbf{Y}_{2}=\mathcal{W}_{2} \widehat{\mathbf{Y}}
\end{array}
$$

Thus we have

$$
\begin{aligned}
\mathbf{X}_{1}^{\dagger} \mathbf{E}_{1}^{-1} \mathbf{X}_{1} & =D\left\{\mathbf{X}_{1}^{\dagger}\left(W_{1} W_{1}^{\dagger}\right)^{-1} \mathbf{X}_{1}\right\} \\
& =D \widehat{\mathbf{X}}^{\dagger} \widehat{\mathbf{X}} \\
& =D \epsilon_{1}
\end{aligned}
$$

$$
\begin{aligned}
\mathbf{X}_{2}^{\dagger} F_{1}^{-1} \mathbf{X}_{2} & =|1-D|\left\{\mathbf{X}_{2}^{\dagger}\left(\mathcal{D}_{1} \mathcal{D}_{1}^{\dagger}\right)^{-1} \mathbf{X}_{2}\right\} \\
& =|1-D| \widehat{\mathbf{Y}}^{\dagger} \widehat{\mathbf{Y}} \\
& =|1-D| \epsilon_{2} \\
\mathbf{Y}_{1}^{\dagger} F_{2}^{-1} Y_{1} & =|1-D|\left\{Y_{1}^{\dagger}\left(\mathcal{D}_{2} \mathcal{D}_{2}^{\dagger}\right)^{-1} \mathbf{Y}_{1}\right\} \\
& =|1-D| \hat{\mathbf{X}}^{\dagger} \widehat{\mathbf{X}} \\
& =|1-D| \epsilon_{\mathbf{1}}
\end{aligned}
$$

and

$$
\begin{aligned}
\underline{\mathbf{Y}}_{2}^{\dagger} \mathrm{E}_{2}^{-1} \mathbf{Y}_{2} & =D\left\{\mathbf{Y}_{2}^{\dagger}\left(W_{2} W_{2}^{\dagger}\right)^{-1} \Psi_{2}\right\} \\
& =D \widehat{\mathbf{Y}^{\dagger}} \\
& =D \epsilon_{2}
\end{aligned}
$$

Here we see that $X_{1}, X_{2}, Y_{1}$ and $Y_{2}$ are each constrained to lie on an ellipse. The motion in each plane is therefore given by the superposition of motion on two ellipses as shown by Ripken. The Courant-Snyder parameters of the ellipses are just the Ripken lattice parameters.

Note that under the difference resonance condition $B=0$ we have, with the help of $(122-123)$ and (132-134),

$$
\begin{aligned}
& \mathcal{D}_{1} \mathcal{D}_{1}^{\dagger}=\frac{1-D}{D} \mathcal{W}_{1} \mathcal{W}_{1}^{\dagger} \\
& \mathcal{D}_{2} \mathcal{D}_{2}^{\dagger}=\frac{1-D}{D} \mathcal{W}_{2} \mathcal{W}_{2}^{\dagger}
\end{aligned}
$$

and it follows that

$$
\mathbf{F}_{1}=\mathbf{E}_{1}, \quad \mathbf{F}_{2}=\mathbf{E}_{2}
$$

Thus in this case the parameters of the two ellipses associated with the horizontal motion are the same and those associated with the vertical motion are the same.

\section{Acknowlegments}

The author thanks Yun Lou for encouragement and a careful reading of the manuscript. Work performed under contract No. DE-AC02-98CH10886 of the U.S. DOE.

\section{A. Appendix}

Suppose that

$$
\mathrm{T}=\mathcal{W} \mathcal{W}^{-1}=\underline{W U N}^{-1}
$$


where $\mathcal{W}$ and $\mathcal{W}$ are symplectic. Multiplying this by $W^{-1}$ from the left and by $\mathcal{W}$ from the right we have

$$
u=W^{-1} W_{W}^{-1} W=\mathcal{O N O}^{-1}
$$

where

$$
o=W^{-1} \underline{w}
$$

Thus

$$
\text { UO }=\mathrm{OU}
$$

and writing

$$
\mathcal{O}=\left(\begin{array}{ll}
\mathbb{P} & \mathbb{V} \\
\mathbb{L} & \mathbb{Q}
\end{array}\right)
$$

where $P, Q, L, V$ are two-by-two matrices, we have

$$
\begin{gathered}
\left(\begin{array}{ll}
\mathcal{A} & 0 \\
0 & \mathcal{B}
\end{array}\right)\left(\begin{array}{ll}
\mathbf{P} & \mathrm{V} \\
\mathrm{L} & \mathbf{Q}
\end{array}\right)= \\
\left(\begin{array}{ll}
\mathbf{P} & \mathbf{V} \\
\mathbf{L} & \mathbf{Q}
\end{array}\right)\left(\begin{array}{ll}
\mathcal{A} & 0 \\
\mathbf{0} & \mathcal{B}
\end{array}\right) .
\end{gathered}
$$

Carrying out the matrix multiplications one then finds

$$
\begin{array}{ll}
\mathcal{A P}=\mathbf{P} \mathcal{A}, & \mathcal{B Q}=\mathbf{Q B} \\
\mathcal{B} \mathbf{L}=\mathbf{L} \mathcal{A}, & \mathcal{A V}=\mathbf{V} \mathcal{B} .
\end{array}
$$

Suppose now that $|\mathbf{L}| \neq 0$. Then we have

$$
\mathcal{B}=\mathbf{L}, \mathcal{A} \mathrm{L}^{-1}
$$

and it follows that $\mathcal{B}$ and $\mathcal{A}$ have the same eigenvalues. This contradicts our assumption that the eigenvalues of $\mathbf{T}$ are distinct. Thus we must have $|\mathbf{L}|=0$. Similarly, one finds that $|\mathbf{V}|=0$. Thus the equations $\mathcal{B L}=\mathrm{L} \mathcal{A}$ and $\mathcal{A V}=\mathrm{VB}$ imply

$$
\begin{array}{ll}
\overline{\mathrm{L}} \mathcal{B L}=0, & \mathbf{L} \mathcal{A} \overline{\mathrm{L}}=0 \\
\overline{\mathrm{V}} \mathcal{A V}=0, & \mathrm{~V} \mathcal{B} \overline{\mathrm{V}}=\mathbf{0}
\end{array}
$$

where

$$
\begin{aligned}
& \mathbf{L}=\left(\begin{array}{ll}
L_{11} & L_{12} \\
L_{21} & L_{22}
\end{array}\right) \\
& \mathbf{V}=\left(\begin{array}{ll}
V_{11} & V_{12} \\
V_{21} & V_{22}
\end{array}\right)
\end{aligned}
$$

and

$$
\mathcal{A}=\left(\begin{array}{rr}
\cos \psi_{1} & \sin \psi_{1} \\
-\sin \psi_{1} & \cos \psi_{1}
\end{array}\right)
$$

$$
\mathcal{B}=\left(\begin{array}{rr}
\cos \psi_{2} & \sin \psi_{2} \\
-\sin \psi_{2} & \cos \psi_{2}
\end{array}\right)
$$

Carrying out the matrix multiplications, one finds

$$
\begin{aligned}
& \left(L_{12}^{2}+L_{22}^{2}\right) \sin \psi_{2}=0 \\
& \left(L_{11}^{2}+L_{21}^{2}\right) \sin \psi_{2}=0 \\
& \left(L_{12}^{2}+L_{11}^{2}\right) \sin \psi_{1}=0 \\
& \left(L_{22}^{2}+L_{21}^{2}\right) \sin \psi_{1}=0
\end{aligned}
$$

with similar equations for the elements of $\mathbf{V}$. Since having both $\sin \psi_{1}=0$ and $\sin \psi_{2}=0$ would contradict our assumption of distinct eigenvalues, at least one of these must be nonzero. It then follows that all elements of $L$ must be zero. The same is true for $\mathbb{V}$. Thus

$$
\mathbb{L}=0, \quad V=0 .
$$

Now, writing out the matrix elements of $P A=A P$ and $\mathbf{Q B}=\mathcal{B} \mathbf{Q}$, one finds

$$
\begin{aligned}
& \left(P_{12}+P_{21}\right) \sin \psi_{1}=0 \\
& \left(P_{11}-P_{22}\right) \sin \psi_{1}=0 \\
& \left(Q_{12}+Q_{21}\right) \sin \psi_{2}=0 \\
& \left(Q_{11}-Q_{22}\right) \sin \psi_{2}=0 .
\end{aligned}
$$

Here our assumption that no eigenvalue is equal to 1 or -1 implies that neither $\sin \psi_{1}$ nor $\sin \psi_{2}$ can be zero. Thus we have

$$
\begin{aligned}
& P_{21}=-P_{12}, \quad P_{22}=P_{11} \\
& Q_{21}=-Q_{12}, \quad Q_{22}=Q_{11} .
\end{aligned}
$$

The matrices $\mathcal{O}, \mathbf{P}$, and $\mathbf{Q}$ therefore must be of the form

$$
\begin{aligned}
& \mathcal{O}=\left(\begin{array}{cc}
\mathbf{P} & 0 \\
0 & \mathbf{Q}
\end{array}\right) \\
& \mathbf{P}=\left(\begin{array}{cc}
C_{1} & S_{1} \\
-S_{1} & C_{1}
\end{array}\right) \\
& \mathbf{Q}=\left(\begin{array}{cc}
C_{2} & S_{2} \\
-S_{2} & C_{2}
\end{array}\right) .
\end{aligned}
$$

Furthermore, since $\mathcal{O}$ is symplectic, we must have

$$
C_{1}^{2}+S_{1}^{2}=1, \quad C_{2}^{2}+S_{2}^{2}=1
$$

and therefore

$$
\mathrm{PP}^{\dagger}=\mathbf{I}, \quad \mathrm{QQ}^{\dagger}=\mathbf{I} . \quad \mathcal{O O}^{\dagger}=\mathbf{I} .
$$




\section{References}

[1] G. Guignard, "The General Theory of All Sum and Difference Resonances in a Three-Dimensional Magnetic Field in a Synchrotron", CERN Report 76-96 (1976).

[2] G. Guignard, "Betatron Coupling and the Related Impact of Radiation", Phys. Rev. E 51, 6104 (1995)

(3) G. Guignard, "Beam Blow-Up and Luminosity Reduction due to Linear Coupling", CERN Report ISR. BOM/77-43 (1977).

[4] D. A. Edwards and L. C. Teng, "Parameterization of Linear Coupled Motion in Periodic Systems", IEEE Trans. on Nucl. Sc. 20, 885 (1973). L. C. Teng, "Concerning n-Dimensional Coupled Motions", NAL Report FN-229, May 3, 1971.

[5] M. Billing, "The Theory of Weakly Coupled Transverse Motion in Storage Rings", Cornell University Report CBN 85-2 (1985).

[6] T. Roser, "Generalized Treatment of Linear Motion in Two-Dimensional Periodic Systems", University of Michigan Report UM-HE 88-8 (1988).

[7] D. Sagan and D. Rubin, "Linear Analysis of Coupled Lattices", Phys. Rev. ST Accel. and Beams 2, 074001 (1999)

[8] G. Ripken and F. Willeke, "Methods of Beam Optics", DESY 88-114, 1988.

[9] F. Willeke and G. Ripken, "Methods of Beam Optics", Physics of Particle Accelerators, AIP Conference Proceedings $184,758-819$ (1989).

[10] E. D. Courant and H. S. Snyder, Annals of Physics 3, $1-48$ (1958).

[11] K. L. Brown and R. V. Servranckx, Particle Accelerators 36, 121-139 (1991).

[12] L. Mirsky, "An Introduction to Linear Algebra", Dover, New York, 1990, pp. 290-296

[13] F. Christoph Iselin, "The MAD Program Physical Methods Manual", CERN, Geneva, September 15, 1994.

[14] Yun Luo, "Linear Coupling Parametrization in the Action-Angle Frame", Phys. Rev. ST Accel. and Beams 7, 124001 (2004)

[15] R. Calaga, R. Tómas and A. Franchi, "Betatron Covpling: Merging Hamiltonian and Matrix Approaches", Phys. Rev. ST Accel. and Beams 8, 034001 (2005)

[16] Yun Luo, "Matrix Perturbation Approach to the Weak Linear Coupling", Nuclear Instruments and Methods in Physics Research A 566, 218-226 (2006).

[17] Yun Luo, et al., "Continuous Measurement of Global Difference Coupling using a Phase-Locked-Loop Tune Meter in the Relativistic Heavy Ion Collider", Phys. Rev. ST Accel. Beams 9, 124001 (2006). 